This is an electronic reprint of the original article. This reprint may differ from the original in pagination and typographic detail.

Author(s): Jodon, R.; Bender, M.; Bennaceur, Karim; Meyer, J.

Title: Constraining the surface properties of effective Skyrme interactions

Year: $\quad 2016$

Version:

Please cite the original version:

Jodon, R., Bender, M., Bennaceur, K., \& Meyer, J. (2016). Constraining the surface properties of effective Skyrme interactions. Physical Review C, 94(2), Article 024335. https://doi.org/10.1103/PhysRevC.94.024335

All material supplied via JYX is protected by copyright and other intellectual property rights, and duplication or sale of all or part of any of the repository collections is not permitted, except that material may be duplicated by you for your research use or educational purposes in electronic or print form. You must obtain permission for any other use. Electronic or print copies may not be offered, whether for sale or otherwise to anyone who is not an authorised user. 


\title{
Constraining the surface properties of effective Skyrme interactions
}

\author{
R. Jodon, ${ }^{1}$ M. Bender, ${ }^{1,2,{ }^{*}}$ K. Bennaceur, ${ }^{1,3,4, \dagger}$ and J. Meyer ${ }^{1, \dagger}$ \\ ${ }^{1} I P N L$, Université de Lyon, Université Lyon 1, CNRS/IN2P3, F-69622 Villeurbanne, France \\ ${ }^{2}$ CENBG, Université Bordeaux 1, CNRS/IN2P3, F-33175 Gradignan, France \\ ${ }^{3}$ Department of Physics, P.O. Box 35 (YFL), University of Jyväskylä, Jyväskylä FI-40014, Finland \\ ${ }^{4}$ Helsinki Institute of Physics, P.O. Box 64, University of Helsinki, Helsinki FI-00014, Finland
}

(Received 4 June 2016; published 25 August 2016)

\begin{abstract}
Background: Deformation energy surfaces map how the total binding energy of a nuclear system depends on the geometrical properties of intrinsic configurations, thereby providing a powerful tool to interpret nuclear spectroscopy and large-amplitude collective-motion phenomena such as fission. The global behavior of the deformation energy is known to be directly connected to the surface properties of the effective interaction used for its calculation.

Purpose: The precise control of surface properties during the parameter adjustment of an effective interaction is key to obtain a reliable and predictive description of nuclear properties. The most relevant indicator is the surface-energy coefficient $a_{\text {surf }}$. There are several possibilities for its definition and estimation, which are not fully equivalent and require a computational effort that can differ by orders of magnitude. The purpose of this study is threefold: first, to identify a scheme for the determination of $a_{\text {surf }}$ that offers the best compromise between robustness, precision, and numerical efficiency; second, to analyze the correlation between values for $a_{\text {surf }}$ and the characteristic energies of the fission barrier of ${ }^{240} \mathrm{Pu}$; and third, to lay out an efficient and robust procedure for how the deformation properties of the Skyrme energy density functional (EDF) can be constrained during the parameter fit.
\end{abstract}

Methods: There are several frequently used possibilities to define and calculate the surface energy coefficient $a_{\text {surf }}$ of effective interactions built for the purpose of self-consistent mean-field calculations. The most direct access is provided by the model system of semi-infinite nuclear matter, but $a_{\text {surf }}$ can also be extracted from the systematics of binding energies of finite nuclei. Calculations can be carried out either self-consistently [Hartree-Fock (HF)], which incorporates quantal shell effects, or in one of the semiclassical extended Thomas-Fermi (ETF) or modified Thomas-Fermi (MTF) approximations. The latter is of particular interest because it provides $a_{\text {surf }}$ as a numerical integral without the need to solve self-consistent equations. Results for semi-infinite nuclear matter obtained with the HF, ETF, and MTF methods will be compared with one another and with $a_{\text {surf }}$, as deduced from ETF calculations of very heavy fictitious nuclei.

Results: The surface energy coefficient of 76 parametrizations of the Skyrme EDF have been calculated. Values obtained with the HF, ETF, and MTF methods are not identical, but differ by fairly constant systematic offsets. By contrast, extracting $a_{\text {surf }}$ from the binding energy of semi-infinite matter or of very large nuclei within the same method gives the same result within the numerical uncertainties.

Conclusions: Despite having some drawbacks compared to the other methods studied here, the MTF approach provides sufficiently precise values for $a_{\text {surf }}$ such that it can be used as a very robust constraint on surface properties during a parameter fit at negligible additional cost. While the excitation energy of superdeformed states and the height of fission barriers is obviously strongly correlated to $a_{\text {surf }}$, the presence of shell effects prevents a one-to-one correspondence between them. As in addition the value of $a_{\text {surf }}$ providing realistic fission barriers depends on the choices made for corrections for spurious motion, its "best value" (within a given scheme to calculate it) depends on the fit protocol. Through the construction of a series of eight parametrizations SLy5s1-SLy5s8 of the standard Skyrme EDF with systematically varied $a_{\text {surf }}$ value, it is shown how to arrive at a fit with realistic deformation properties.

DOI: 10.1103/PhysRevC.94.024335

\section{INTRODUCTION}

Energy density functional (EDF) methods are versatile tools for the study of nuclear structure and dynamics [1]. Once a parametrization of the EDF has been constructed by selecting terms that incorporate the relevant degrees of

\footnotetext{
*bender@ipnl.in2p3.fr

†bennaceur@ipnl.in2p3.fr

${ }^{\ddagger}$ jmeyer@ipnl.in2p3.fr
}

freedom and by carefully fixing each term's coefficient, it can be applied to nuclei throughout the chart of nuclei at the level of static or time-dependent mean-field calculations, random phase approximation (RPA) and its extensions, or for the description of large-amplitude motion in the context of the generator coordinate method.

One popular example is the widely used Skyrme EDF [1-4]. Its further improvement is presently pushed into two major directions. One concerns the protocol for the adjustment of its parameters, where the number and diversity of data and pseudodata considered during the fit are increased and various 
postfit analyses added that allow for the quantification of confidence intervals of model parameters and the estimate of statistical error bars [5-10]. The other is the setup of more general forms of the Skyrme EDF containing higher-order terms with additional parameters [11-17]. Because both developments substantially increase the numerical cost of the parameters' adjustment, it is of advantage to have efficient methods to calculate the data and pseudodata used during the fit.

In the past, many authors have discussed the crucial role that an accurate adjustment of the surface energy coefficient of an EDF's parametrization plays for the deformation properties of nuclei [7,9,18-22]. Generally speaking, when increasing $a_{\text {surf }}$, deformation energy surfaces become stiffer, fission barriers higher, and the excitation energy of fission isomers and other superdeformed states more elevated. This, in turn can then be used to fine tune the EDF. First pioneering calculations of fission properties indicated that the two early Skyrme parametrizations SIII [23] and SkM [24] give a fission barrier height for ${ }^{240} \mathrm{Pu}$ that is too high or too low by roughly a factor of two, respectively. This disagreement clearly exhibited within semiclassical calculations [25] has triggered the adjustment of the $\mathrm{SkM}^{*}$ parametrization, for which the parameters of the momentum-dependent part of SkM have been modified and fine-tuned to change the height of the semiclassical fission barrier without changing other properties of infinite nuclear matter [18]. Even after more than 30 years, $\mathrm{SkM}^{*}$ is a still-often-used reference for the description of fission phenomena [26-31].

The aim of the present study is threefold. First, we set up and benchmark an efficient and robust method to calculate the surface energy coefficient $a_{\text {surf }}$ as defined in Ref. [32] for modern Skyrme EDFs. Second, we analyze the correlation between the value for $a_{\text {surf }}$ and characteristic energies of the deformation energy landscape of ${ }^{240} \mathrm{Pu}$. Third, we outline how a constraint on the value of $a_{\text {surf }}$ can be incorporated into the parameter adjustment.

This article is organized as follows. In Sec. II we present the three methods based on a one-dimensional model for semi-infinite nuclear matter that will be used to evaluate the surface energy coefficient of the Skyrme EDF: the selfconsistent Hartree-Fock method (HF), the extended ThomasFermi method (ETF), and the modified Thomas-Fermi method (MTF). Section III recalls the "leptodermous protocol" of Reinhard et al. [33] to extract the surface energy from an analysis of very large fictitious nuclei. We use this approach with the ETF method. A systematic comparison of the results provided by these four possibilities is presented in Sec. IV. In Sec. V, we discuss the extent to which the so-extracted surface energy coefficient is linked to characteristic energies of the fission barrier of ${ }^{240} \mathrm{Pu}$. Finally, Sec. VI presents the adjustment of a series of standard Skyrme parametrizations with systematically varied $a_{\text {surf }}$, called SLy5s1-SLy5s8. A summary of our discussion and perspectives is given in Sec. VII.

\section{SURFACE ENERGY IN SEMI-INFINITE NUCLEAR MATTER}

\section{A. The Skyrme EDF}

The starting point for the derivation of a Skyrme EDF is usually an effective two-body interaction with parameters that may depend on the density of the system. It is important to recall that this effective interaction is used only as a generator for the general form of the EDF and allows for writing the coupling constants that weight these scalars as functions of a limited number of parameters. It is then common to disregard certain terms in the functional or to relax some of the interdependencies between the coupling constants, which breaks the one-to-one correspondence between the EDF and the underlying effective interaction used to construct it $[1,11,17,34]$.

The present standard form of the Skyrme EDF is motivated by the use of a density-dependent two-body interaction [1], leading to a bilinear EDF with density-dependent coupling constants. Most of the parametrizations discussed below are of that type. However, we also compare with results obtained for EDFs derived from interactions with two-, three-, and sometimes even four-body terms but density-independent coupling constants $[16,17,35]$. Such functionals are referred to as trilinear or quartic EDFs, respectively.

In general, the total energy can be written as the sum of five terms [1]: the kinetic energy $E_{\text {kin }}$, a potential energy functional $E_{\text {Sky }}$ that models the strong interaction in the particle-hole channel, a pairing energy functional $E_{\text {pairing, a }}$ Coulomb energy functional $E_{\text {Coulomb }}$, and a correction term $E_{\text {corr }}$ that approximately removes the excitation energy owing to spurious motions caused by broken symmetries,

$$
E=E_{\text {kin }}+E_{\text {Sky }}+E_{\text {pairing }}+E_{\text {Coulomb }}+E_{\text {corr }} \text {. }
$$

The model systems that we use to extract the surface properties are semi-infinite nuclear matter and giant spherical nuclei, for which only the first two parts of the energy are taken into account. As a consequence of the Skyrme interaction being a contact force, the corresponding EDF then can be written in the form of an integral over a local energy density,

$$
\begin{aligned}
E & =\int d^{3} r \mathcal{E}(\mathbf{r}), \\
\mathcal{E}(\mathbf{r}) & =\mathcal{E}_{\text {kin }}(\mathbf{r})+\mathcal{E}_{\mathrm{Sky}}(\mathbf{r}),
\end{aligned}
$$

where the Skyrme part $\mathcal{E}_{\text {Sky }}(\mathbf{r})$ can be further decomposed into central $\left(\mathcal{E}_{t}^{\mathrm{C}}\right)$, spin-orbit $\left(\mathcal{E}_{t}^{\mathrm{LS}}\right)$, and tensor $\left(\mathcal{E}_{t}^{\mathrm{T}}\right)$ terms,

$$
\mathcal{E}_{\mathrm{Sky}}(\mathbf{r})=\sum_{t=0,1}\left[\mathcal{E}_{t}^{\mathrm{C}}(\mathbf{r})+\mathcal{E}_{t}^{\mathrm{LS}}(\mathbf{r})+\mathcal{E}_{t}^{\mathrm{T}}(\mathbf{r})\right],
$$

that are either composed entirely of isoscalar densities $(t=0)$ or that contain bilinear combinations of isovector densities $(t=1)$. The Skyrme energy functional, as such, is constructed to be an isoscalar.

The physics contained in the Skyrme functional has been discussed in great detail in the literature [1,11,17,34,36], and here we use standard notations for local densities and coefficients of the functional. Its complete form discussed in these papers contains many terms, only a small subset of which are present for the systems we consider here. In particular, all time-odd densities are zero because of the time-reversal invariance we impose, and many components of the time-even vector and tensor densities are zero because of spatial symmetries. 


\section{B. Model of semi-infinite nuclear matter}

The surface energy is often extracted from an idealized onedimensional model of semi-infinite nuclear matter originally developed by Swiatecki [37] and revisited in Ref. [32]. One considers a medium where the local densities are constant along the $x$ and $y$ directions, but vary along the $z$ direction. The corresponding profile of the local proton and neutron matter densities is noted as $\rho_{q}(z), q=n, p$. Deep inside the matter for $z \rightarrow-\infty$, one expects that $\rho_{0}(z) \rightarrow \rho_{\text {sat }}$, i.e., the equilibrium density of infinite nuclear matter, and that $\mathcal{E}(z) \rightarrow a_{v}$, i.e., the volume energy per particle at saturation. Far outside the matter, i.e., for $z \rightarrow+\infty$, one has $\rho_{0}(z) \rightarrow 0$. For the sake of compact notation, we drop the $z$ dependence of the densities from here on whenever possible.

Within this one-dimensional model, the only nonvanishing components of the Cartesian spin-current tensor density $J_{0, \mu v}$ are $J_{0, x y}=-J_{0, y x}$. As a consequence, only the $J_{0, z}$ component of the vector part $\mathbf{J}_{0}$ of the full spin-current tensor as defined in Refs. [11,36] is nonzero.

We here focus on the discussion of the surface energy coefficient $a_{\text {surf }}$ that is related to the surface energy of symmetric semi-infinite nuclear matter. In this system, proton and neutron densities are equal; i.e., $\rho_{n}(z)=\rho_{p}(z)=\frac{1}{2} \rho_{0}(z)$, and similar for the other densities. As a consequence, only those terms in the EDF that are entirely composed of isoscalar densities have to be considered. This enormously simplifies the energy density (2) that can be reduced to ${ }^{1}$

$$
\begin{aligned}
\mathcal{E}= & \frac{\hbar^{2}}{2 m_{0}^{*}\left[\rho_{0}\right]} \tau_{0}+C_{0}^{\rho}\left[\rho_{0}\right] \rho_{0}^{2}-C_{0}^{\Delta \rho}\left(\nabla \rho_{0}\right)^{2} \\
& +\frac{1}{2} C_{0}^{J} \mathbf{J}_{0}^{2}-C_{0}^{\nabla J} \mathbf{J}_{0} \cdot \nabla \rho_{0} \\
& +B_{0}^{\rho} \rho_{0}^{3}+B_{0}^{\nabla \rho} \rho_{0}\left(\nabla \rho_{0}\right)^{2}+\frac{1}{2} B_{0}^{J} \mathbf{J}_{0}^{2} \rho+D_{0}^{\rho} \rho_{0}^{4},
\end{aligned}
$$

where the density-dependent isoscalar effective mass is given by the ratio

$$
\frac{m}{m_{0}^{*}\left[\rho_{0}\right]}=1+\frac{2 m}{\hbar^{2}}\left(C_{0}^{\tau} \rho_{0}+B_{0}^{\tau} \rho_{0}^{2}\right) .
$$

The expression for the Skyrme EDF provided by Eq. (5) covers a vast number of different parametrizations that have been lately used in the literature. We use a notation where the coupling constants for the bilinear, trilinear, and quartic terms are denoted with $C_{0}, B_{0}$, and $D_{0}$, respectively.

In the most widely used standard form of the Skyrme EDF only the bilinear terms are considered, i.e., all $B_{0}=$ $D_{0}=0$, and the coupling constant $C_{0}^{\rho}$ is made explicitly density dependent by multiplying it with $\left[1+c \rho_{0}^{\alpha}(\mathbf{r})\right]$, where the parameter $c$ controls the relative weight of the densitydependent part of the coupling constant.

The $\mathbf{J}_{0}^{2}$ terms bilinear in the spin-current density are called "tensor terms" in what follows. For a majority of the widely used parametrizations the coupling constant $C_{0}^{J}$ of the tensor

\footnotetext{
${ }^{1}$ Note that no unique definition of the coupling constant $C_{t}^{J}$ of the tensor terms can be found in the literature. We use here the convention of Ref. [11]. Others might differ by a factor two.
}

terms is set to zero, such that $\mathcal{E}_{t}^{\mathrm{T}}=0$ in Eq. (4). Many past semiclassical and HF calculations of semi-infinite nuclear matter, however, have neglected these terms also for those parametrizations for which they are to be taken into account.

The possibility of replacing the density dependence of $C_{0}^{\rho}$ with trilinear $\left(B_{0} \neq 0\right)$ and quartic $\left(D_{0} \neq 0\right)$ terms, and where all coupling constants are derived from an underlying Skyrme $2+3+4$-body Hamiltonian, has been considered recently with the goal of constructing well-defined EDFs for use in beyond-mean-field methods [16,17]. This extended form of the Skyrme EDF is also considered in the present work.

The assumptions made when setting up the Skyrme EDF have been discussed in great detail in the literature $[1,4,11,17,34,36]$ and are not recapitulated here. Neither do we repeat the discussion concerning the different possible definitions for the surface energy. For a detailed discussion, we refer to the original articles of Myers and Swiatecki [38,39] and the more recent ones by Pearson et al. [40,41], Brack et al. [25], Kolehmainen et al. [42], Centelles et al. [43], and Douchin et al. [44].

\section{Hartree-Fock calculations}

The first one of the methods we use to calculate semi-infinite nuclear matter is the self-consistent mean-field approximation, usually called HF, with a treatment along the lines of Refs. [32,40]. In this case, one considers the quantity [32]

$$
E_{L}=\int_{-L}^{+L} d z \mathcal{E}(z)
$$

which represents the energy per unit of surface for a piece of semi-infinite nuclear matter described by a density which is constant in the $x$ and $y$ directions and extends from $-L$ to $+L$ in the $z$ direction with the conditions $\rho_{0}(-L)=\rho_{\text {sat }}$ and $\rho_{0}(L)=0$ for $L \rightarrow+\infty$. After solving the mean-field equations, the surface energy coefficient, denoted as $a_{\text {surf }}^{\mathrm{HF}}$, can be extracted using

$$
a_{\mathrm{surf}}^{\mathrm{HF}}=\lim _{L \rightarrow \infty} 4 \pi r_{0}^{2} \int_{-L}^{+L} d z\left[\mathcal{E}(z)-a_{v} \rho_{0}(z)\right]
$$

with the parameter $r_{0}$ being defined through the condition $\frac{4}{3} \pi r_{0}^{3} \rho_{\text {sat }}=1$.

As a particular feature of such quantal calculation, one observes so-called "Friedel oscillations" $[45,46]$ of the density $\rho_{0}(z)$ in the vicinity of the surface inside the matter. Because these oscillations are only very slowly damped, a reliable calculation of $a_{\text {surf }}^{\mathrm{HF}}$ requires quite large an interval in the $z$ direction.

Our HF values for $a_{\text {surf }}$ often differ slightly from those given by other groups in the past [18,47-51]; see the Supplemental Material [52]. On the one hand, this underlines the numerical difficulties of determining a precise value for $a_{\text {surf }}$. On the other hand, as said before, the contribution from the $\mathbf{J}^{2}$ tensor terms, which are present for a subset of the parametrizations, has been omitted in most of the earlier published work. 


\section{Semiclassical calculations}

As a second method to determine $a_{\text {surf }}$ we use the semiclassical ETF approach up to order $\hbar^{4}$ [25]. Values obtained with this method are denoted as $a_{\text {surf }}^{\mathrm{ETF}}$ in what follows.

In this semiclassical framework, the local densities $\rho_{q}(z)$ are modeled by a three-parameter modified Fermi function. The kinetic and spin-current densities entering the total energy density $\mathcal{E}(z)$ are obtained from an expansion of the so-called single-particle Bloch density matrix in powers of $\hbar$ around its Thomas-Fermi value as originally proposed by Wigner and Kirkwood. In short, the Bloch density matrix is the coordinatespace representation of the statistical operator $e^{\beta \hat{h}}$ constructed from the HF single-particle Hamiltonian $\hat{h}$ expressed in its eigenbasis [53] and related to the usual coordinate-space representation of the density matrix $\rho\left(\mathbf{r}, \mathbf{r}^{\prime}\right)$ through a Laplace transform [25,53]. Ultimately, this leads to expressions for $\tau_{0}$ and $\mathbf{J}_{0}$ as functions of the local density $\rho_{0}$ and its derivatives, where it is customary to separate the contributions of different (even) power in $\hbar$ in the expansion

$$
\begin{aligned}
\tau_{0} & =\tau_{0}^{[0]}+\tau_{0}^{[2]}+\tau_{0}^{[4]}, \\
\mathbf{J}_{0} & =\mathbf{J}_{0}^{[2]}+\mathbf{J}_{0}^{[4]} .
\end{aligned}
$$

The complete expressions for $\tau_{0}^{[0]}$ (which is simply the kinetic energy of a noninteracting Fermi gas), $\tau_{0}^{[2]}, \tau_{0}^{[4]}, \mathbf{J}_{0}^{[2]}$, and $\mathbf{J}_{0}^{[4]}$, have been given by Brack et al. [25] and more recently by Bartel et al. [54]. Note that, in the case where tensor terms are included in the Skyrme EDF, the standard expressions given in the early articles have to be modified taking into account the results of Bartel et al. [55] for the contribution of the $\mathbf{J}^{2}$ terms. Minimizing the surface energy as calculated with an expression equal to the one given by Eq. (8), one obtains the parameters of the assumed Fermi-type density profiles from which the surface energy coefficients can then be calculated.

\section{E. Modified Thomas-Fermi approximation}

The ETF approximation provides expressions for the kinetic and spin-current densities in terms of the nucleon density and its derivatives. The MTF approximation developed by Krivine and Treiner [56] consists in using an ETF expansion limited to order $\hbar^{2}$, where the coefficients are modified to simulate the order $\hbar^{4}$ as well as the $\hbar^{2}$ effective mass contributions. The MTF form of the kinetic and spin-current densities can then be written as

$$
\begin{aligned}
\tau_{0}^{[2]} & =\alpha k_{F}^{2} \rho_{0}+\beta \frac{\left(\nabla \rho_{0}\right)^{2}}{\rho_{0}}+\gamma \Delta \rho_{0}+\tau_{0}^{[2(\mathrm{so})]}, \\
\tau_{0}^{[2(\mathrm{so})]} & =\frac{1}{2}\left(\frac{2 m_{0}^{*}\left[\rho_{0}\right]}{\hbar^{2}} \mathbf{W}_{0}\right)^{2} \rho_{0}, \\
\mathbf{J}_{0}^{[2]} & =-\frac{2 m_{0}^{*}\left[\rho_{0}\right]}{\hbar^{2}} \rho_{0} \mathbf{W}_{0},
\end{aligned}
$$

with the coefficients $\alpha=\frac{3}{5}, \beta=\frac{1}{18}$, and $\gamma=\frac{1}{3}$, chosen for a reasonable reproduction of the total energy of finite nuclei [56]. In local density approximation, the Fermi momentum is given by $k_{F}=\left(\frac{3}{2} \pi^{2} \rho_{0}\right)^{1 / 3}$ and the spin-orbit field $\mathbf{W}_{0}$ is defined as usual as $\mathbf{W}_{0}=\partial \mathcal{E} / \partial \mathbf{J}_{0}$.
The coefficients $\alpha$ and $\gamma$ of the MTF expressions thus keep their values obtained from ETF, whereas the value of $\beta$ is changed from $1 / 36$ (obtained within ETF) to 1/18 (used in the MTF method).

The interest of the MTF method is that in the case of symmetric semi-infinite matter, the approximations provided by Eqs. (11), (12), and (13) lead to an analytically solvable Euler-Lagrange equation for the density profile without the need for carrying out a variational calculation numerically. It is noteworthy that the entire density profile is varied in the MTF method, whereas in the ETF approach only the parameters of a predefined Fermi function are optimized to give the lowest binding energy.

Following the derivation outlined in Ref. [57], the semiclassical energy density of symmetric matter can then be simply written as the sum of two terms,

$$
\mathcal{E}=h_{v}\left[\rho_{0}\right]+h_{s}\left[\rho_{0}\right] \frac{\left(\nabla \rho_{0}\right)^{2}}{\rho_{0}}
$$

where

$$
\begin{aligned}
& h_{v}\left[\rho_{0}\right]=C_{0}^{\rho} \rho_{0}^{2}+B_{0}^{\rho} \rho_{0}^{3}+D_{0}^{\rho} \rho_{0}^{4}+\alpha \frac{\hbar^{2}}{2 m_{0}^{*}} k_{F}^{2} \rho_{0}, \\
& h_{s}\left[\rho_{0}\right]=\frac{\hbar^{2}}{2 m} \beta+d \rho_{0}+g \rho_{0}^{2}+V_{\mathrm{so}}\left[\rho_{0}\right] \rho_{0}^{2},
\end{aligned}
$$

with

$$
\begin{aligned}
d & =(\beta-\gamma) C_{0}^{\tau}-C_{0}^{\Delta \rho}, \\
g & =(\beta-2 \gamma) B_{0}^{\tau}+B_{0}^{\nabla \rho}, \\
V_{\text {so }}[\rho] & =-\frac{1}{2} \frac{\left(C_{0}^{\nabla J}\right)^{2}}{Q\left[\rho_{0}\right]} .
\end{aligned}
$$

Compared to what is found elsewhere in the literature, the expressions (15) and (16) have been complemented to take into account the possible three- and four-body contributions to the Skyrme EDF (5), as introduced in Refs. [16,17]. In addition, to take into account the tensor terms in the Skyrme EDF, the vector spin-current density is obtained through the protocol of Bartel et al. [55], which leads to a redefinition of the $V_{\text {so }}[\rho]$ term,

$$
\begin{aligned}
\mathbf{J}^{[2]} & =\frac{C_{0}^{\nabla J}}{Q\left[\rho_{0}\right]} \nabla \rho_{0}, \\
Q\left[\rho_{0}\right] & =\frac{\hbar^{2}}{2 m_{0}^{*}\left[\rho_{0}\right]}+C_{0}^{J} \rho_{0}+B_{0}^{J} \rho_{0}^{2},
\end{aligned}
$$

which, compared to the original work by Treiner and Krivine [57], contains additional terms.

Inserting the analytical solution for the density profile $\rho_{0}(z)$ into the expression for the surface energy, one obtains after some further analytical manipulations a compact expression for the surface energy coefficient (for details, see Ref. [57]), denoted $a_{\text {surf }}^{\mathrm{MTF}}$ from here on,

$$
a_{\text {surf }}^{\mathrm{MTF}}=8 \pi r_{0}^{2} \int_{0}^{\rho_{0}} d \rho\left\{h_{s}[\rho]\left[\frac{E}{A}(\rho)-\frac{E}{A}\left(\rho_{\text {sat }}\right)\right]\right\}^{\frac{1}{2}} .
$$

Because the MTF method differs from ETF approach only by the values of the coefficients, this expression has exactly 
the same form as the Wilets formula [58,59] derived much earlier. Hereafter, we call Eq. (22) the MTF pocket formula to underline the simplicity of its use to calculate $a_{\text {surf }}$ compared to the HF and ETF methods described above.

In its original MTF form without the contribution from the tensor terms, this expression has been occasionally used to analyze the surface properties of Skyrme parametrizations $[57,60,61]$.

\section{SURFACE ENERGY FROM SEMICLASSICAL CALCULATIONS OF LARGE NUCLEI}

In Ref. [33], Reinhard et al. proposed a protocol to extract the nuclear liquid-drop coefficients that correspond to an EDF parametrization from a leptodermous expansion based on mean-field calculations of very large fictitious spherical nuclei calculated without Coulomb interaction and pairing correlations. For the determination of the (isoscalar) surface energy coefficient, the calculations can be limited to symmetric nuclei with $N=Z=A / 2$. In this case, the calculated binding energy of the finite nuclei $E(A)$ can then be developed into

$$
E(A) \approx a_{\mathrm{vol}} A+a_{\mathrm{surf}} A^{2 / 3}+a_{\mathrm{curv}} A^{1 / 3}+a_{0} A^{0},
$$

i.e., a volume, surface, and curvature term plus another one that is proportional to $A^{0}=1$. The latter was not considered in Ref. [33]. Despite its unrealistic limit for $A \rightarrow 0$, such a term emerges naturally in the leptodermous expansion of the nuclear binding energy as a second-order correction to the curvature energy $[62,63]$ or when replacing the geometric surface energy of the standard liquid-drop model by a double-folding integral $[64,65]$ as it is done in the finite-range liquid-drop and droplet models (FRLDMs) [66].

For consistency with semi-infinite matter calculations, the finite nuclei are calculated without center-of-mass correction, irrespective of the scheme used during the fit of the parametrization used.

To extract the surface energy coefficient $a_{\text {surf }}$ of Eq. (23), one first defines an effective surface energy coefficient $a_{\text {surf }}^{\text {eff }}(A)$ of a given nucleus of mass $A$ by reshuffling the expansion (23)

$$
\begin{aligned}
a_{\text {surf }}^{\text {eff }}(A) & \equiv\left[\frac{E(A)}{A}-a_{\mathrm{vol}}\right] A^{1 / 3} \\
& =a_{\text {surf }}+a_{\text {curv }} A^{-1 / 3}+a_{0} A^{-2 / 3} .
\end{aligned}
$$

The volume energy coefficient $a_{\mathrm{vol}}$ is provided by the energy per particle $E / A$ of symmetric infinite nuclear matter at saturation density. By fitting a second-order polynomial in $A^{-1 / 3}$ to the calculated values for $a_{\text {surf }}^{\text {eff }}(A)$, one obtains the coefficients $a_{\text {surf }}, a_{\text {curv }}$, and $a_{0}$.

To disentangle the surface energy unambiguously from the higher-order terms in the liquid-drop formula (23), we calculate 20 nuclei with very large mass numbers in the range $1200 \leqslant A \leqslant 200000$, similar to what was done in Ref. [33]. There, however, nuclei were calculated self-consistently, which required to remove shell effects by subtracting the shell correction as obtained from the self-consistent single-particle spectrum from the total binding energy. Here we use the semiclassical ETF approach to calculate binding energies instead, such that there is no need to eliminate shell effects.
The expansion in terms of powers of $A^{-1 / 3}$ (or inverse nuclear radius) is thus more stable and the LDM parameters thus more precisely extracted when extrapolating to (semi-)infinite nuclear matter in the limit $A^{-1 / 3} \rightarrow 0$. The so-determined values for $a_{\text {surf }}$, however, should be compared to the ETF results obtained for semi-infinite-matter and not the HF results as the ones extracted in Ref. [33].

\section{DETERMINATION OF $\boldsymbol{a}_{\text {surf }}$}

In this section we present a systematic comparison of results for the surface energy coefficient obtained with the four aforementioned methods. One of our main goals is to check whether the computationally friendly MTF pocket formula provides a reliable estimate for the surface energy coefficient as extracted from the theoretically more advanced, but numerically more involved, HF or ETF methods. For that purpose, we use a large set of Skyrme parametrizations.

\section{A. Parametrizations considered}

There is a large number of Skyrme parametrizations that can be found in the literature. Only few of them, however, are frequently used in production runs. The simplicity and popularity of the Skyrme EDF has led to quite large a number of "experimental" fits of parametrizations that were carried out for one or the other very specific study. In particular, there are many "families" of fits that explore the influence of variations of details of the parametrizations on their predictive power. We profit here from this large number of parametrizations as it allows us to cover large intervals of values for infinite nuclear-matter properties, which in turn can reveal possible correlations of differences between the four methods to determine $a_{\text {surf }}$ and other global features of the parametrizations.

Here we give a brief overview over the main features and particularities of the parametrizations considered here (which are all of the standard density-dependent bilinear form unless specified otherwise).

(i) SIII [23]. The coupling constants $C_{t}^{\rho}$ of this early, but still sometimes used, parametrization are linear functions of the density $\rho_{0}$. Ska [67], SGI, SGII [68], SkM [24], SkM* [18]. These are examples of early standard parametrizations with a density dependence with $\alpha<1$, taking values of either $1 / 3$ or $1 / 6$ as almost all parametrizations listed below, unless otherwise specified.

(iii) The SLy family of fits. More recent and widely used examples of standard parametrizations are the Saclay-Lyon fits SLy4-SLy7 [69] that differ in options for center-of-mass correction and tensor terms, SLy5* [70], a recent refit that suppresses the finite-size spin instability of the original SLy5 parametrization, and SLy4d [71], a refit of SLy4 without any center-of-mass correction built for the purpose of time-dependent Hartree-Fock (TDHF) calculations.

(iv) The TIJ family of fits [11]. For these parametrizations, also fitted within the Saclay-Lyon protocol, 
the coupling constants of the tensor terms were systematically varied over large intervals.

(v) SLy5 + T [72], SLy4T, SLy4T min $_{\text {[73], SLy4T }}$ self, TZA [74]. These are further fits based on the SaclayLyon family of fits with either added or modified tensor terms.

(vi) $f_{0}$ and $f_{ \pm}[75]$. These constitute another series of variants of Saclay-Lyon parametrizations that explore different values for the splitting of the effective mass of protons and neutrons in asymmetric matter. Unlike the other standard parametrizations considered here, their coupling constants $C_{t}^{\rho}$ have two density dependencies with powers $\alpha_{1}=1 / 3$ and $\alpha_{2}=2 / 3$, respectively. This allows for the decoupling of nuclear-matter properties that cannot be chosen independently for standard parametrizations with just one density dependence [76].

(vii) The SLyIII. $x$ family of fits [77]. These are yet another series of variants of Saclay-Lyon parametrizations that were built specifically for the purpose of regularized beyond-mean-field calculations. All have the same linear density dependence of the $C_{t}^{\rho}$ coupling constant as SIII. Their isoscalar effective mass $m_{0}^{*} / m=x$ has been constrained to the values $0.7,0.8,0.9$, and 1.0 in units of the nucleon mass.

(viii) SaMi [78]. This recent parametrization has been adjusted within a SLy-inspired protocol with the aim of improved spin-isospin properties.

(ix) $\mathrm{SkI} 3, \mathrm{SkI} 4$ [79], SkO, $\mathrm{SkO}^{\prime}$ [80]. These fits are examples of standard parametrizations with a generalized isospin dependence of the spin-orbit part of the EDF.

(x) The SV family of fits [81]. This series of parametrizations was constructed for the purpose of studying the correlation of nuclear-matter properties with other observables. Starting from the reference parametrization called SV-bas, nine others were constructed by varying the incompressibility $K_{\infty}$, isoscalar effective mass $m_{0}^{*} / m$, symmetry energy $J$, and the sum-rule enhancement factor $\kappa_{v}$ one by one while keeping the others constant. A best fit, called SV-min, is also considered.

(xi) The UNEDF family of fits. These parametrizations differ by the selection of data considered in the fit protocol. Compared to UNEDF0 [6], for example, UNEDF1 [7] and UNEDF2 [9] are also adjusted to reproduce the excitation energy of the fission isomer of ${ }^{240} \mathrm{Pu}$. We also consider the parametrization $\mathrm{UNEDF}{ }^{\text {so }}$ [82] that corresponds to a readjustment of the spin-orbit coupling constants of UNEDF1, thereby improving the spectroscopy of very heavy nuclei at the expense of a much lower value for $a_{\text {surf }}$.

(xii) The BSk family of fits. We also consider several representative parametrizations from the series of large-scale Bruxelles-Montreal Skyrme-HartreeFock-Bogoliubov mass fits that stay within the standard form of the Skyrme EDF with only the coupling constants $C_{t}^{\rho}$ being density dependent
[21,49,83-88], among which BSk14 [21] has also been fitted to fission barriers.

(xiii) LNS [89], NRAPR [90], NRAPRii [91]. In one way or another, these parametrizations of the standard Skyrme EDF were adjusted to reproduce nuclear-matter properties as predicted by ab initio methods. While LNS was adjusted to reproduce a large variety of nuclear-matter results from a Brueckner-HF calculation, NRAPR has been fitted to the density dependence of the energy per particle as obtained from ab initio calculations of nuclear and neutron matter. We also consider the parametrization NRAPRii with doubled strength of the spin-orbit interaction compared to NRAPR, as suggested by the authors of Ref. [91].

(xiv) KDE0v1 [61]. This standard parametrization has been adjusted to reproduce a large number of empirical nuclear-matter data.

(xv) SQMC700 [92]. This parametrization of the standard Skyrme EDF has been derived as the nonrelativistic mean-field limit of a quark-meson-coupling model [92].

(xvi) S1Sd, S1Se [93]. The parameters of these two standard density-dependent Skyrme parametrizations have been adjusted to reproduce total binding energies of doubly magic nuclei as predicted by the Gogny force D1S. For S1Sd the tensor terms were included, whereas for S1Se they were neglected.

(xvii) S3Ly family of fits [35]. We also include a few representative examples from the series of fits of extended Skyrme EDFs that add central threebody terms with gradients to a standard densitydependent two-body Skyrme EDF and which were carried out within a modified Saclay-Lyon fit protocol. These fits do systematically cover a wide range of values for the isoscalar effective mass $m_{0}^{*} / m$ and incompressibility $K_{\infty}$. For example, S3Ly71260 is a parametrization with $m^{*} / m_{0}=0.71$ and $K_{\infty}=$ $260 \mathrm{MeV}$.

(xviii) SLyMR0 [16], SLyMR1 [94]. We also include two recent parametrizations built for the purpose of spuriousity-free beyond-mean-field calculations: SLyMR0, which combines the non-densitydependent part of the standard two-body central and spin-orbit Skyrme interaction with gradientless three- and four-body terms, and SLyMR1, where the four-body terms are replaced with the three-body terms with gradients as introduced in Ref. [17].

The main interest of the four rarely used parametrizations KDE0v1, LNS, NRAPRii, and SQMC700 is that they were recently shown to be consistent with a large set of pseudodata for infinite symmetric nuclear matter [95]. Their predictive power for finite nuclei, however, is rather limited [91]. Most, if not all, other parametrizations of the standard density-dependent Skyrme EDF listed above provide a much better description of finite nuclei than these, but in turn are incompatible with some of the presently accepted values for the empirical properties of 
nuclear matter [95]. It should be stressed, however, that none of the nuclear-matter properties is a real observable, as in one way or the other they all have to be extracted in a model-dependent way from data.

\section{B. Surface-energy coefficients}

Figure 1 shows the surface energy coefficients $a_{\text {surf }}$ obtained from the HF, ETF, and MTF methods for the list of parametrizations given above. Two sets of ETF values, one obtained from the calculation of semi-infinite nuclear matter (open blue squares) and the other extracted from calculations of large finite nuclei (blue dots), are shown.

The first observation that can be made is that the values of the surface energy coefficient spread over a relatively large interval, from about 15.5 to about $19.5 \mathrm{MeV}$ for the results given by the HF calculations. As many parametrizations were obtained within dissimilar protocols, it is difficult to correlate the value for $a_{\text {surf }}$ with other properties of the respective parametrizations. A few correlations that can be unambiguously identified are that $a_{\text {surf }}$ depends on the presence and size of tensor terms (compare SLy4, SLy5, SLy5T, and the TIJ), the size of the spin-orbit term (compare NRAPR and NRAPRii), and the scheme for center-of-mass correction used (as already pointed out in [20]; compare SLy4, SLy6, and SLy4d). One can expect that there are further correlations between a parametrization's value for $a_{\text {surf }}$ and its other properties. The analysis of their origin and nature, i.e., if they are a physical necessity or rather an accidental consequence of either a specific fit protocol or an overconstrained form of the EDF, however, is beyond the scope of the present study.

A second observation is that the differences between the values for $a_{\text {surf }}^{\mathrm{HF}}$ and $a_{\mathrm{surf}}^{\mathrm{ETF}}$, on the one hand, and the differences between $a_{\text {surf }}^{\mathrm{HF}}$ and $a_{\text {surf }}^{\mathrm{MTF}}$, on the other hand, are fairly constant and almost independent on the nature of the EDF considered (density-dependent bilinear, trilinear, or even quartic) and the properties it provides for infinite nuclear matter.

A third observation is that for all parametrizations the discrepancy between the two different ETF values is so small that it can be hardly resolved on the figure, which confirms that the leptodermous protocol of Ref. [33] offers a reliable alternative to calculations of semi-infinite nuclear matter, with the remaining differences being on the order of $10 \mathrm{keV}$.

The size and parametrization dependence of the little remaining scatter between the three methods to determine $a_{\text {surf }}$ from semi-infinite nuclear matter calculations can be better resolved on Fig. 2, where the differences $\Delta a_{\text {surf }}^{\mathrm{HF}-\mathrm{ETF}} \equiv$ $a_{\text {surf }}^{\mathrm{HF}}-a_{\text {surf }}^{\mathrm{ETF}}$ and $\Delta a_{\text {surf }}^{\mathrm{HF}-\mathrm{MTF}} \equiv a_{\text {surf }}^{\mathrm{HF}}-a_{\text {surf }}^{\mathrm{MTF}}$ are directly plotted for the same sample of parametrizations as in Fig. 1. For most parametrizations, $\Delta a_{\text {surf }}^{\mathrm{HF}-\mathrm{ETF}}$ is close to $+0.5 \mathrm{MeV}$ and $\Delta a_{\text {surf }}^{\text {HF-MTF }}$ is close to $-0.5 \mathrm{MeV}$. Nonetheless, in some cases the differences can deviate from this global trend; especially for $\Delta a_{\text {surf }}^{\text {HF-MTF }}$ one can find values in the range between $-1 \mathrm{MeV}$ and about zero. In any event, Fig. 2 indicates that, for the purpose of calculating $a_{\text {surf }}$, MTF is almost as good an approximation to HF as ETF. However, the two semiclassical methods differ among each other on a much larger scale as one systematically overestimates the HF value, whereas the other systematically underestimates it.

A closer examination of the parametrizations for which the scatter is largest indicates that $\Delta a_{\text {surf }}^{\mathrm{HF}-\mathrm{MTF}}$ may strongly depend on the isoscalar effective mass; see, for example, the SLyIII. $x$ and BSk series for which $m_{0}^{*} / m$ ranges between 0.7 to 1.05 .

In some cases one also finds small differences between parametrizations with similar effective mass that are correlated
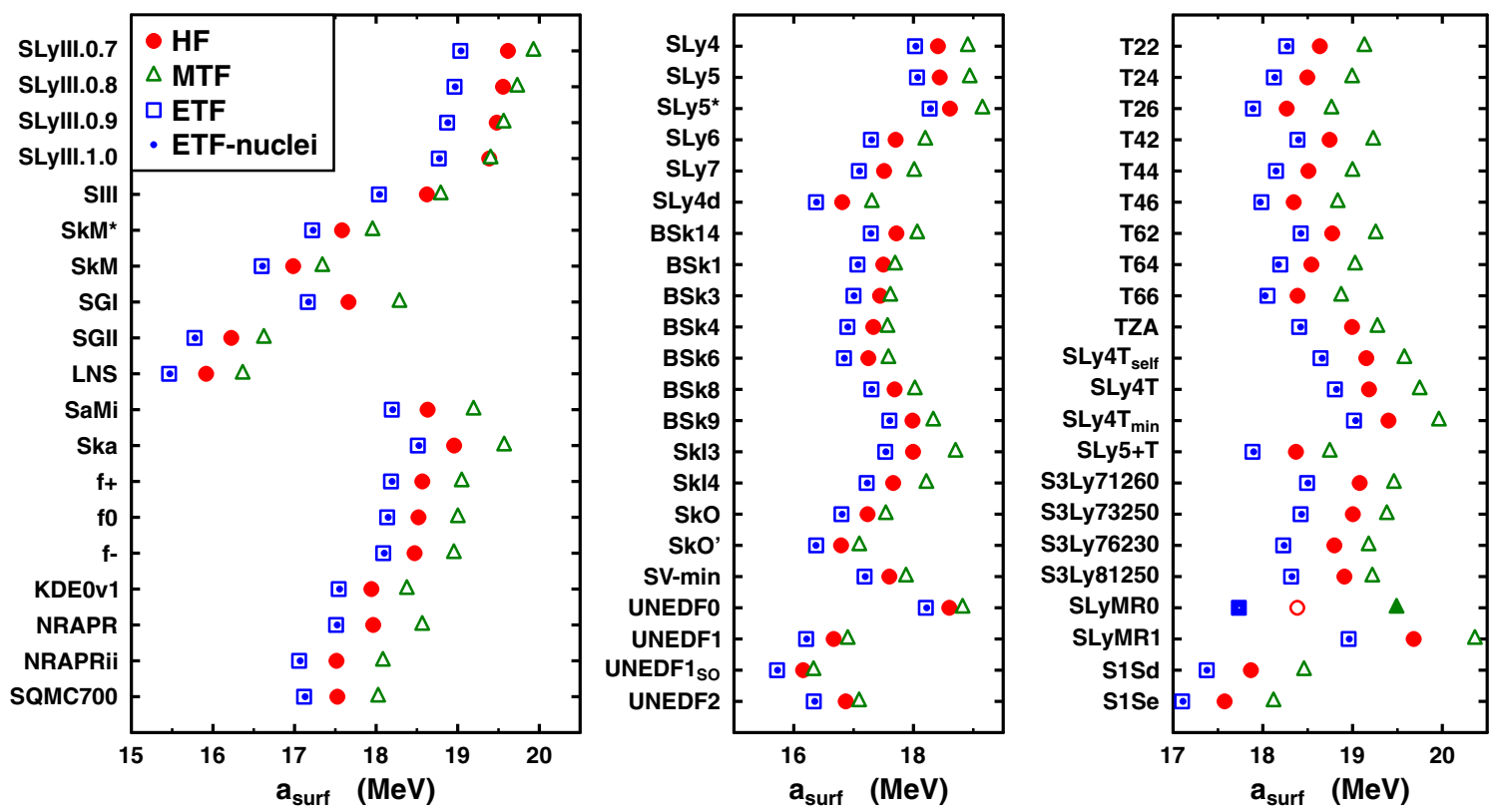

FIG. 1. Surface-energy coefficients obtained within HF, MTF, and ETF approaches for a large set of Skyrme parametrizations (see text). ETF values are determined from semi-infinite matter calculations and extracted from calculations of finite nuclei, as explained in Sec. III. Note that all three panels share the same energy scale despite covering different intervals. Results for the SLyMR0 parametrization (inverted markers) have been artificially increased by three $\mathrm{MeV}$ to remain within the range of the figure. 

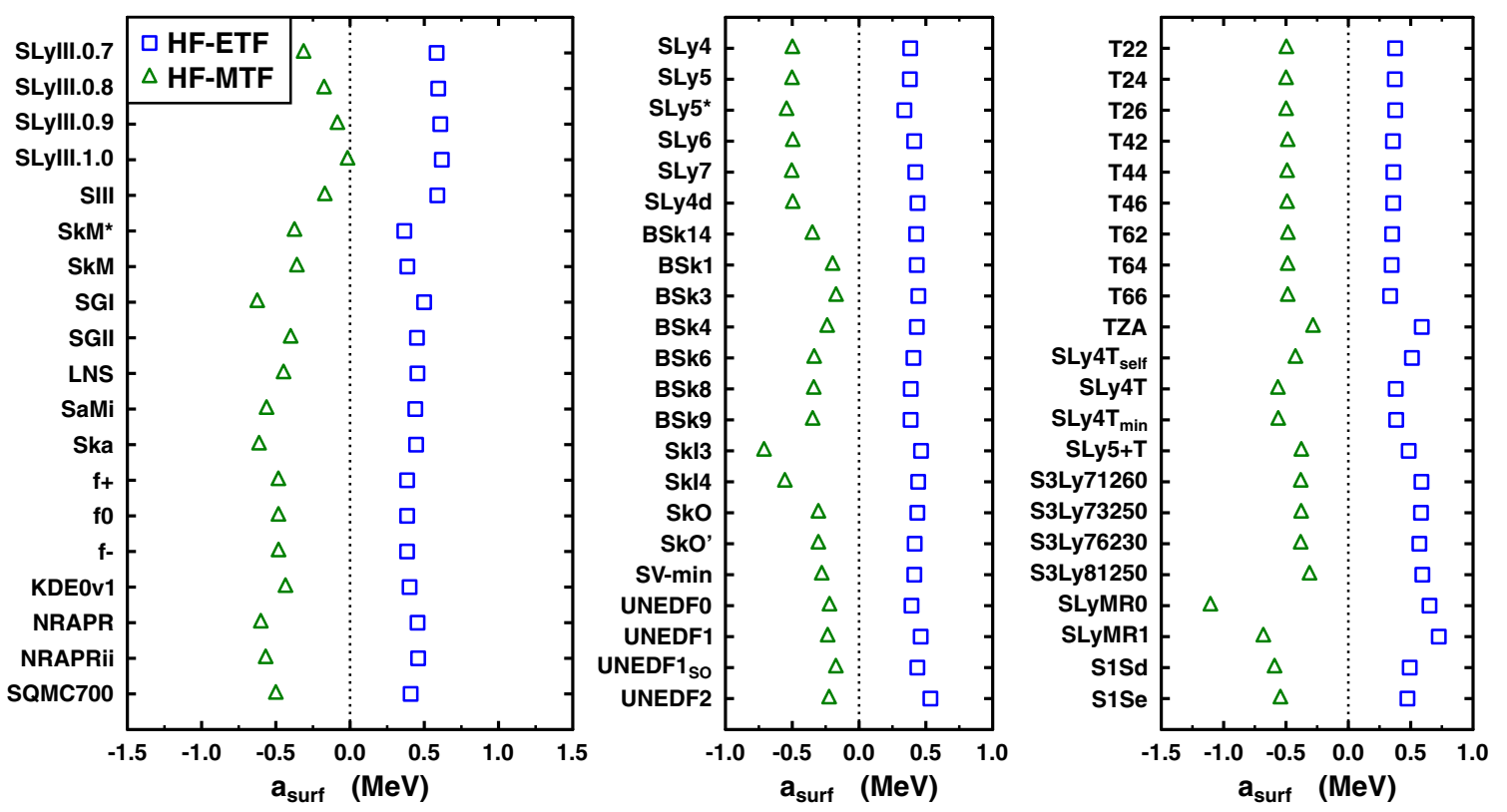

FIG. 2. Differences between the surface energy coefficients obtained with the HF, ETF, and MTF models for the same parametrizations as in Fig. 1.

to the strength of the tensor terms. This is exemplified by the comparison of SLy4, SLy5, SLy5 + T, and the TIJ series, which were adjusted within almost the same protocol, have practically the same effective mass, but differ in the size and sign of the tensor coupling constants $C_{t}^{J}$. For these cases the difference between ETF and MTF seems not to be much affected, but that the differences between HF and ETF and also between HF and MTF are not the same. In general, Fig. 2 suggests that without tensor terms, ETF and MTF have the same offset from HF, but in opposite directions. In the presence of tensor terms, the ETF values for $a_{\text {surf }}$ come closer to the ones from HF, whereas MTF moves further away. This finding points to limitations of modeling the spin-current density in semiclassical methods.

Comparing SLy5 and SLyIII.0.7, which have similar effective mass and tensor terms, indicates that $\Delta a_{\text {surf }}^{\mathrm{HF}-\mathrm{MTF}}$ might in addition also depend on the power of the density dependence of $C_{0}^{\rho}\left[\rho_{0}\right]$. For $\alpha=1$ (SLyIII.0.7), MTF values for $a_{\text {surf }}$ are much closer to the ones from HF than for $\alpha=1 / 6$ (SLy5). We come back to this below.

One can expect that there might be further weak correlations between a parametrization's value for $\Delta a_{\text {surf }}^{\mathrm{HF} \text { MTF }}$ and its other properties, but these cannot easily be identified even within this large set of parametrizations.

To analyze further the correlation between $\Delta a_{\text {surf }}^{\mathrm{HF}}$ MTF and nuclear-matter properties, parametrizations with systematically varied nuclear-matter properties are needed. Such fits are provided by the series of SV parametrizations by Klüpfel et al. [81]. During their adjustment, the incompressibility $K_{\infty}$, isoscalar effective mass $m_{0}^{*} / m$, symmetry energy coefficient $J$, and the Thomas-Reiche-Kuhn enhancement factor $\kappa_{v}$ have been separately varied while keeping the other properties constant.

The left panel of Fig. 3 shows the corresponding values for $a_{\text {surf }}$ obtained with the four schemes to calculate the surface en- ergy coefficient introduced above. Two correlations for the size of $a_{\text {sym }}$ itself, independent of the method of its determination, become immediately obvious: $a_{\text {surf }}$ increases with increasing incompressibility $K_{\infty}$ and also with increasing symmetry energy coefficient $J$. Both, however, might be particular to the specific fit protocol of this series of parametrizations.

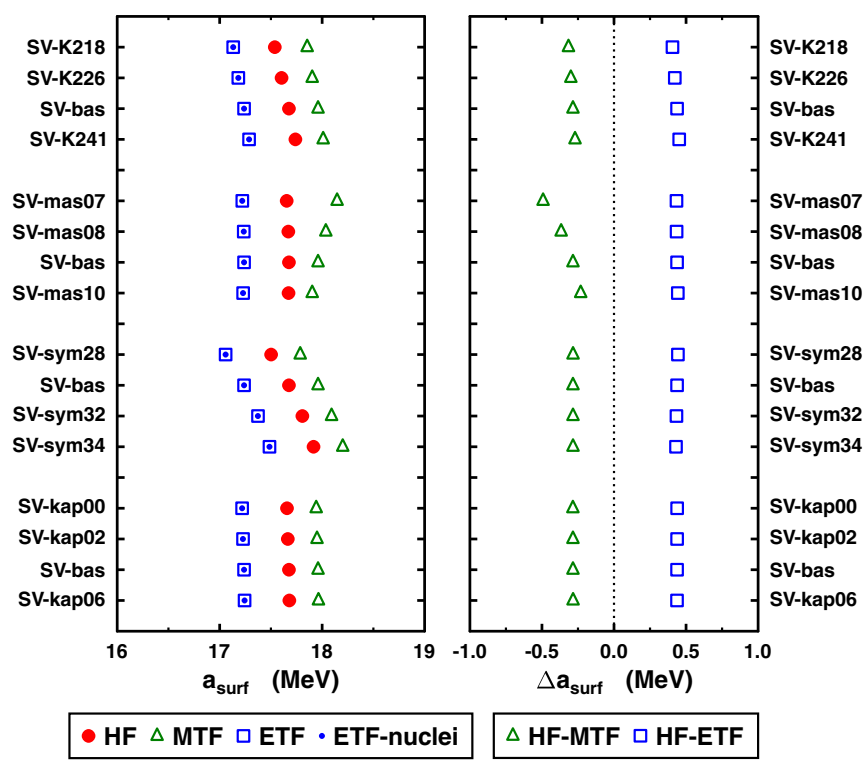

FIG. 3. (Left) Surface energy coefficients obtained within the HF, MTF, and ETF approach for the family of SV parametrizations of Ref. [81]. Each of the four series systematically varies one bulk property (from top to bottom: $K_{\infty}, m_{0}^{*} / m, J, \kappa_{v}$ ) around the value of SV-bas while keeping the others constant. (Right) Same as Fig. 2, but for the series of SV fits. 

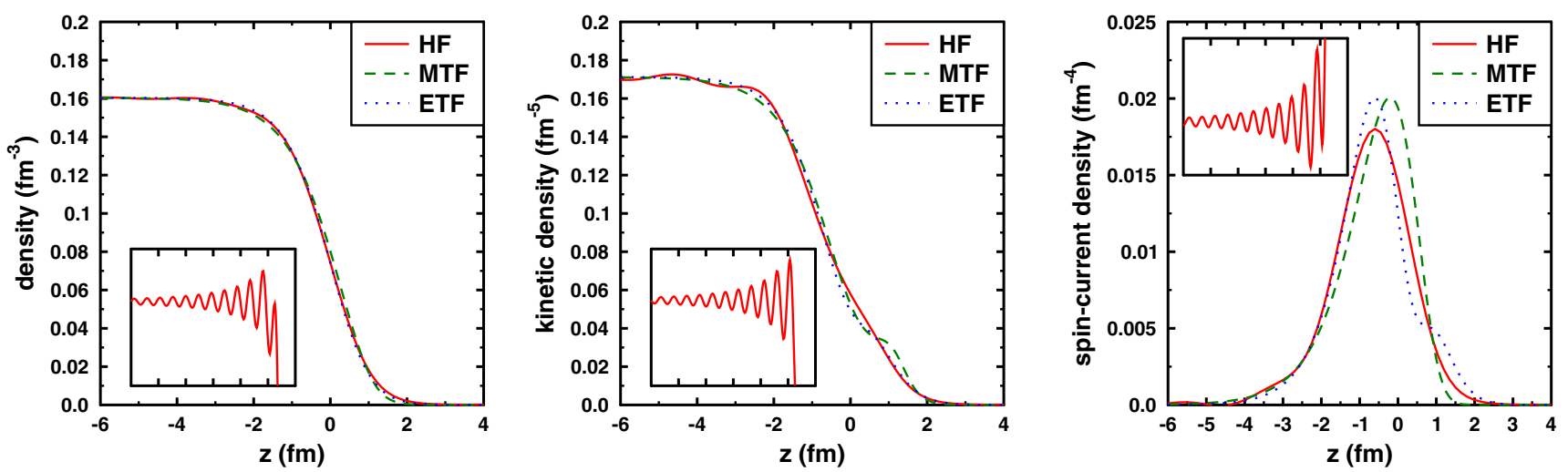

FIG. 4. Radial profile of the mass density $\rho(z)$, kinetic density $\tau(z)$, and the $z$ component of the spin-current density $J_{z}(z)$ as obtained from HF, ETF, and MTF calculations of semi-infinite nuclear matter with the SLy5 parametrization. The inserts give an enlarged view of the Friedel oscillations of the HF densities inside the matter in an interval of $30 \mathrm{fm}$ below the surface.

More importantly, for the series with varied effective mass $m_{0}^{*} / m$, the different methods to determine $a_{\text {surf }}$ give a different trend, as already indicated by the analysis of the large set of parametrizations in Fig. 2. While the HF and ETF values are fairly independent on $m_{0}^{*} / m$, the MTF values rapidly increase with decreasing effective mass. The method dependence of the $a_{\text {surf }}$ values becomes more obvious in Fig. 3, where are plotted the differences between the methods in the same way as in Fig. 2.

The right panel of Fig. 3 also reveals that there is a slight dependence of both $\Delta a_{\text {surf }}^{\text {HF-ETF }}$ and $\Delta a_{\text {surf }}^{\text {HF-MTF }}$ on the incompressibility $K_{\infty}$, which to a large extent explains the difference of $\Delta a_{\text {surf }}^{\mathrm{HF}-\mathrm{MTF}}$ between SLy5 and SLyIII.0.7 found in Fig. 2 and attributed to the power of the densitydependent term above. The values for $K_{\infty}$ of these two parametrizations are quite different, $K_{\infty}=230 \mathrm{MeV}$ for SLy5 and $K_{\infty}=361.3 \mathrm{MeV}$ for SLyIII.0.7, which is a consequence of the correlation between $m_{0}^{*} / m, K_{\infty}$, and the power of the density dependence of the $C_{0}^{\rho}$ coupling constant analyzed, for example, in Ref. [76]. The $K_{\infty}$ value of SLyIII.0.7 is far outside the range covered by the SV parametrizations of Fig. 3 (and therefore also far from the empirical value, which is unavoidable for standard parametrizations with linear density dependence [76]). Going from SV-K218 to SV-K241, one finds that $\Delta a_{\text {surf }}^{\mathrm{HF}}$ the same slope when going from SLy5 to SLyIII.0.7, their $\Delta a_{\text {surf }}^{\mathrm{HF}-\mathrm{MTF}}$ should differ by about $260 \mathrm{keV}$, which is indeed the case.

Altogether, we find a reasonably parametrizationindependent behavior of the three models with a quantitative difference that is almost constant as long as $K_{\infty}$ and $m_{0}^{*} / m$ are constrained to a small interval, which is usually the case within a given fit protocol. From this, one can conclude that the Wilets pocket formula from Eq. (22) can be safely used in a fit protocol to determine the coupling constants of future Skyrme EDFs.

\section{Density profiles in semi-infinite nuclear matter}

To examine the origin of the systematic differences found above between the three models used to calculate semi-infinite nuclear matter, Fig. 4 displays the radial profiles of the mass density $\rho(z)$, kinetic density $\tau(z)$, and the $z$ component of the spin-current density $J_{z}(z)$, as obtained from each method.

For each model, the profiles are positioned such that the lower boundary $-L^{\prime}$ of a piece of the surface with some sufficiently large, but otherwise arbitrarily chosen, particle number $A$ calculated as

$$
A=4 \pi r_{0}^{2} \int_{-L^{\prime}}^{\infty} d z \rho(z)
$$

coincides for all of the three models. ${ }^{2}$ The parameter $r_{0}$ is the same as in Eq. (8). With this, the density profiles can be compared exactly as those of finite nuclei with same particle number. The origin $z=0$, however, has been chosen to correspond to the position of the sharp surface of a piece of saturated nuclear matter with constant density inside that is placed in precisely the same manner as the three density profiles shown in Fig. 4 , i.e., $-L^{\prime}=A /\left(4 \pi r_{0}^{2} \rho_{\text {sat }}\right)=$ $A /\left[3^{1 / 3}\left(4 \pi \rho_{\text {sat }}\right)^{2 / 3}\right]$.

As for finite nuclei, the density and kinetic density are bulk properties that approach a saturation value inside the matter and fall off at the surface. By contrast, the spin-current density is peaked on the surface and approaches zero inside and outside the matter. Indeed, $J_{z}$ is exactly zero in infinite homogeneous nuclear matter when calculated in mean-field approximation.

The amplitude of the Friedel oscillations exhibited by all three HF densities is comparatively small and barely visible when plotting the entire density profile. However, as indicated by the inserts, the oscillations with a wavelength of about $2.4 \mathrm{fm}$ are only slowly damped and reach far inside.

\footnotetext{
${ }^{2}$ None of our three codes for HF, ETF, or MTF calculations of semi-infinite nuclear matter constrains the particle number in the integration interval. Still, within each model, the value of $-L^{\prime}$ can be easily determined from the interpolation of $A(L)$ obtained from semi-infinite-matter calculations with systematically varied intervals $[-L,+L]$ in Eq. (7), etc. Indeed, for sufficiently large intervals, $A$ becomes a linear function of $L$. The value $A=40$ has been chosen to prepare Fig. 4.
} 
Each of the three approaches to calculate semi-infinite matter provides a slightly different profile of the mass density distribution $\rho(z)$ at the surface. They differ in the steepness, diffuseness, and also in the position of the inflection point. This is not very surprising, as in one way or the other the ETF and MTF methods are constructed to provide an approximation to the total energy of an HF calculation and not to reproduce its densities. While in the ETF method only the parameters of a Fermi function parametrizing the profile of the local matter density $\rho(z)$ are variationally optimized, the entire density profile is implicitly varied in the MTF approach.

The ETF density $\rho(z)$ follows quite closely the mean of the oscillations of the HF density, such that the two are difficult to distinguish in Fig. 4. By contrast, the profile of the MTF density is visibly different from the other two by being much more asymmetric around the surface. Inside the matter, the MTF density approaches the saturation value slower than the ETF density or the mean of the oscillating HF density, whereas outside the matter it falls much more quickly to zero than the other two. The same behavior has already been found for finite nuclei in the seminal papers on the MTF method [56,57]. This asymmetry has some consequences for the corresponding kinetic and spin-current densities. On the one hand, it generates a visible bump in the tail of $\tau(z)$ and also leads to a very asymmetric shape of $J_{z}(z)$.

A similar, but much less pronounced, bump is also found in the tail of the ETF kinetic density $\tau(z)$. In this case, it is generated by the terms of order $\hbar^{4}$ in the semiclassical expansion, which are also at the origin of the bump that the ETF spin-current density $J_{z}(z)$ exhibits at the same position. The appearance and size of the latter is parametrization dependent, and can be correlated to the relative sign and size of spin-orbit and tensor terms.

While these differences in the density profiles easily explain why the three methods deliver slightly different results for the surface energy coefficient, it is more difficult to correlate the dissimilarities of the density profiles with the systematic differences found for the $a_{\text {surf }}$ values. In any event, it has to be recalled that the ETF and MTF approaches are approximations to the HF calculation of the total energy of the nuclear system. The surface energy coefficient defined through Eq. (8), however, is the difference between two energies that are typically two orders of magnitude larger, such that the differences found between the various schemes to calculate them are beyond the third significant digit, which should not be unexpected.

\section{RELATION BETWEEN $a_{\text {surf AND DEFORMATION }}$ ENERGY SURFACES}

It is well understood that the characteristic features of the deformation energy surfaces of heavy nuclei, i.e., the excitation energies of secondary minima and the height of barriers separating the minima and stabilizing the nucleus against fission, are strongly correlated with the surface properties of the effective interaction $[18,22]$. An emblematic example is the "double-humped" fission barrier of ${ }^{240} \mathrm{Pu}$ [96] that we use here as an illustrative example. As a rule of thumb, the larger the surface energy coefficient $a_{\text {surf }}$, the higher the excitation energies and barriers.

In the liquid-drop model, the surface energy of a nucleus is simply provided by the product of $a_{\text {surf }}$, the size of the nucleus' surface and a universal factor. Assuming that the deformation of the fission isomer and the top of the barriers turn out to be at the same deformation for all parametrizations (such that the nuclear surface is of comparable size), one would then naively expect that their energy is linearly correlated with the surface energy coefficient. In addition, the larger the deformation, the steeper should be the slope.

It has to be recalled, however, that the characteristic features of the energy surfaces, in particular the ground-state deformation and the presence of secondary minima, are caused by shell effects $[96,97]$. As a consequence, the variation of shell effects with deformation is as important for the observable excitation energies as is the smooth variation of the of the liquid-drop surface energy with deformation, which on its own would just give all actinide nuclei a spherical ground state and one structureless broad fission barrier. Indeed, the fact that the amplitude of the variation of shell effects with deformation that leads to the characteristic double-humped fission barrier of most actinides is correlated with the effective mass and spin-orbit strength has been pointed out already very early by Tondeur [98].

For a subset of the parametrizations employed above, we have carried out calculations of the complete static fission barrier of ${ }^{240} \mathrm{Pu}$, which is an often-used benchmark for such studies [1,18-20,28,99-102]. To avoid a readjustment of the pairing strength and the ambiguities related to it, we have limited the analysis to parametrizations of similar effective mass. Most were adjusted with a variant of the Saclay-Lyon protocol. In the figures we only distinguish between series of fits, which are the SLyx family, (SLy4-7, SLy5*, SLy4d), the fx family $\left(\mathrm{f}_{0}, \mathrm{f}_{ \pm}\right)$, the TIJ family (T22, T24, T26, T42, T44, T46, T62, T64), and the SLyxT family (SLy5+T, SLy4T, $\left.\mathrm{SLy}_{4} \mathrm{~T}_{\text {min }}, \mathrm{SLy}_{4} \mathrm{~T}_{\text {self }}, \mathrm{TZA}\right)$. In addition, we have used the classic SkM and SkM* parametrizations. As it turns out, this subset is sufficient for a conclusive analysis.

In all cases, we use "surface pairing" with strength $-1250 \mathrm{MeV} \mathrm{fm}^{-3}$ for protons and neutrons, and a soft cutoff at $\pm 5 \mathrm{MeV}$ above and below the Fermi energy as defined in Ref. [103].

Calculations are carried out with the most recent versions of the EV8 [34] and EV4 [104] codes. Both use the same three-dimensional coordinate-space representation, where the single-particle wave functions are discretized on an equidistant mesh in a box. The two codes differ by the symmetries they impose on the nuclear shapes. EV8 assumes three plane reflection symmetries, which reduces the calculation to $1 / 8$ of the full box, but is still sufficient to describe triaxial shapes. By contrast, EV4 assumes only two plane reflection symmetries, which then also permits to describe (not necessarily axial) octupole deformed shapes. The EV8 box has $n_{x} \times n_{y} \times n_{z}=$ $20 \times 20 \times 30$ points of distance $0.8 \mathrm{fm}$. The Poisson equation for the Coulomb field is solved in a $50 \times 50 \times 50$ box for improved precision of the Coulomb energy at large elongation. The EV4 box doubles the $z$ dimension. With these choices, 


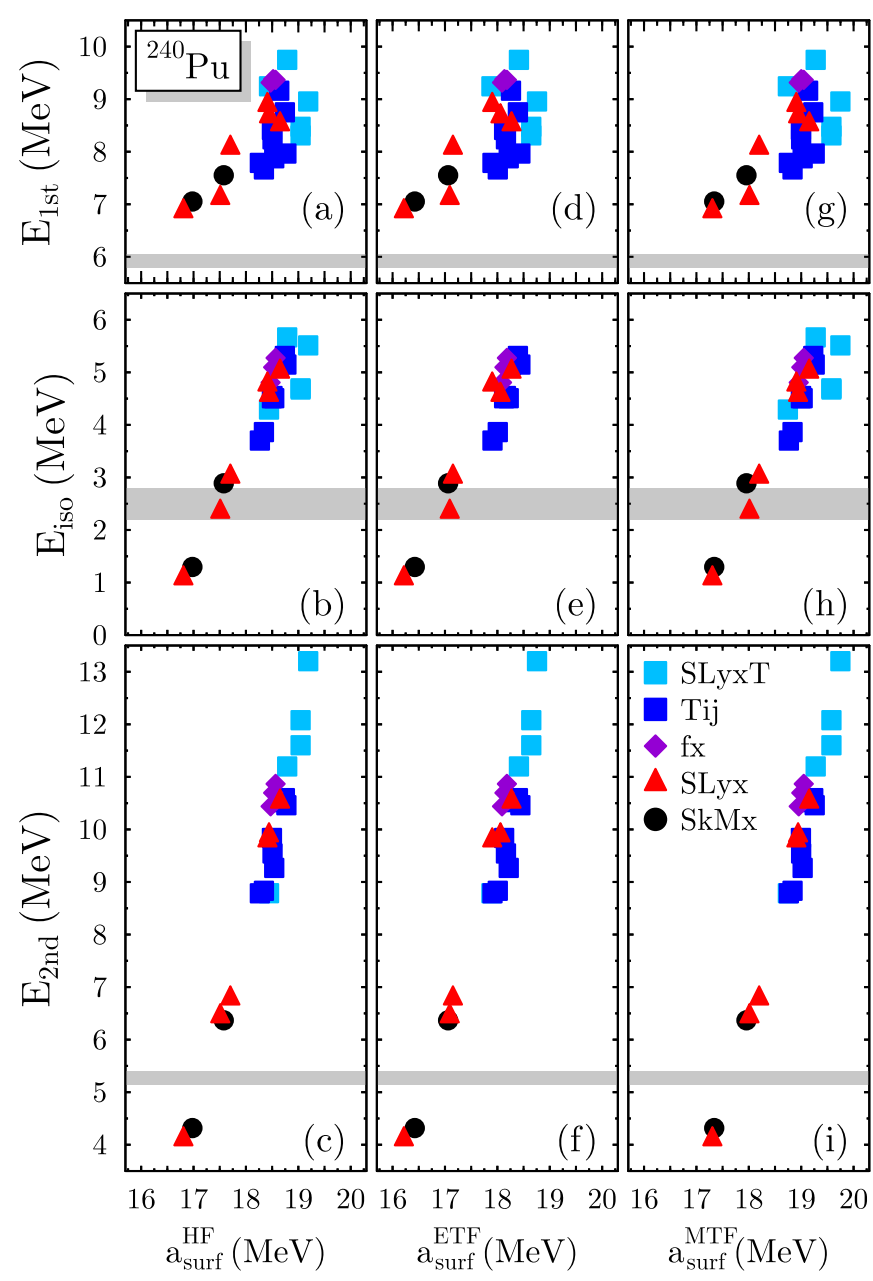

FIG. 5. Correlation between the excitation energy of the fission isomer (middle panels), the height of the inner (top panels) and outer (bottom panels) barrier of ${ }^{240} \mathrm{Pu}$, and the surface energy coefficient $a_{\text {surf }}$ calculated in HF (left column), ETF (middle column), and MTF (right column) for a selection of Skyrme parametrizations (see text). The horizontal gray bars indicate the range of experimental data found in the literature (see text). Note that all panels share the same energy scale.

the deformation energy reaches a precision of about $100 \mathrm{keV}$ independent on the nuclear shape [105].

Up to the fission isomer, the shapes along the static fission path are reflection symmetric, whereas beyond the fission isomer the shapes quickly become mass asymmetric with increasing quadrupole deformation. The inner barrier is triaxial; everywhere else the shapes along the path are axial.

Figure 5 shows the excitation energy of the superdeformed fission isomer and the height of the inner and outer barriers as a function of the surface energy coefficient calculated in three different ways.

For the experimental energies, which are indicated by gray horizontal bars in Fig. 5, slightly conflicting values have been reported in the literature. For the excitation energy of the isomer one finds $2.4 \pm 0.3 \mathrm{MeV}$ [96], $\approx 2.8 \mathrm{MeV}$ [106], and $2.25 \pm 0.20 \mathrm{MeV}$ [107]. For the height of the barriers, the compilation of Ref. [96] lists 5.95 MeV for the inner one and $\approx 5.4 \mathrm{MeV}$ for the outer one. According to the compilation cited by Mamdouh et al. [108], the first barrier has $5.8 \mathrm{MeV}$ and the second barrier $5.3 \mathrm{MeV}$. A more recent paper from the same group [49] cites $6.1 \mathrm{MeV}$ for the inner barrier and $5.2 \mathrm{MeV}$ for the outer barrier. In their paper on the fit of UNEDF1, Kortelainen et al. [7] cite the values $6.05 \mathrm{MeV}$ for the inner barrier and $5.15 \mathrm{MeV}$ for the outer one, as recommended by the RIPL-3 database $[109,110]$. The scatter of the experimental data, however, is much smaller than the scatter in the theoretical results of Fig. 5. In any event, most parametrizations largely overestimate the experimental values, which is the principal motivation for the current efforts to improve the EDFs in that respect.

Note that none of the parametrizations does simultaneously describe all of the three properties. The three parametrizations that give a reasonable description of $E_{\text {iso }}$ of about $2.7 \mathrm{MeV}$ (and which are SkM*, SLy6, and SLy7) still overestimate the inner and outer barrier heights by more than $1 \mathrm{MeV}$.

All deformation energies increase with the value of the surface energy coefficient $a_{\text {surf }}$ as expected. However, the correlations are not strictly linear. Instead, there is a large scatter around the global trends, in particular for the height of the inner barrier. This is an immediate consequence of the shell effects not being the same for all parametrizations. Indeed, it has been demonstrated in Ref. [22] that the contribution of the shell correction as deduced from self-consistent mean-field calculations of the excitation energy of the fission isomer of nuclei in the actinide region, including ${ }^{240} \mathrm{Pu}$, can vary by more than $1 \mathrm{MeV}$ when going from one parametrization to another. The parametrization dependence of shell effects becomes obvious when one directly compares the entire barriers, as can be seen from Fig. 6. Even the overall shape of the barrier is not the same for all parametrizations. The deformation of the fission isomer varies, as does the deformation of the configuration corresponding to the top of the barriers. Even more intriguingly, for some parametrizations there appears a third minimum at large asymmetric shapes, or the topography around the spherical point is quite different. For the purpose of the present paper, it is not important to disentangle the origin of these variations, which are, for example, related to the strength of spin-orbit and tensor terms as will be discussed in a forthcoming article. What is relevant is that there obviously is a large variation of the deformation dependence of the shell effects among the parametrizations studied here. This, in turn, indicates that the adjustment of the excitation energy of the fission isomer or the fission barrier heights cannot be easily replaced by an adjustment of a universal empirical value of the surface energy coefficient.

This is complicated further by the apparent impossibility of determining a precise value for $a_{\text {surf }}$ in a model-independent way. As indicated by the analyses of Refs. [10,33], a reliable extraction of $a_{\text {surf }}$ and other liquid-drop parameters from microscopically calculated binding energies of finite nuclei requires to go to systems with $A \approx 10^{5}$ nucleons. The same can be expected to hold for its reliable extraction from the binding energies of real nuclei, but the systems needed do not exist in nature. Second, the preferred value for $a_{\text {surf }}$ will also depend on choices made for quantum corrections to the binding energy in a given fit. For example, the size of the rotational 


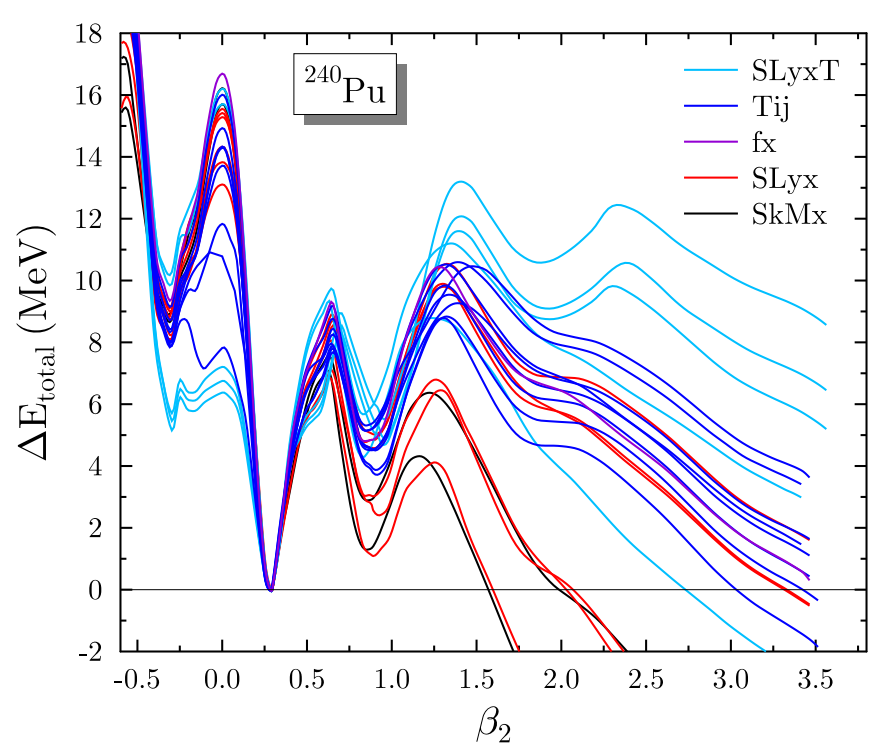

FIG. 6. Deformation energy curve of ${ }^{240} \mathrm{Pu}$ as a function of the dimensionless mass quadrupole moment $\beta_{2}=\frac{4 \pi}{3 R_{0}^{2} A}\left\langle\hat{Q}_{20}\right\rangle$, where $R_{0}=1.2 A^{1 / 3} \mathrm{fm}$ and $A$ is the mass number, for the same set of parametrizations as in Fig. 5 and using the same color code for the families of parametrizations. All deformation energies are normalized to the respective ground-state energy. At large deformation, the curves end where the calculations jump from a solution with large elongation to a solution with two separate fragments.

correction increases rapidly with deformation $[1,21,99]$, such that parametrizations that are supposed to be used with it require a larger value for $a_{\text {surf }}$ than parametrizations supposed to be used without it. Also, for parametrizations that are supposed to be used in beyond-mean-field models, it is the energy difference between collective states that should be compared with data [99], not the difference of minima of the energy surface.

\section{A FIT PROTOCOL INCLUDING $\boldsymbol{a}_{\text {surf }}$}

In one way or another, information about fission barriers has already been sometimes used to constrain parametrizations of effective interactions of Skyrme [7,18,19,21] type.

Using the fission barriers themselves for the parameter adjustment, however, is quite costly. Assuming that shell effects are principally fixed by the other ingredients of the fit protocol, our results suggest a way to fit the information contained in fission barriers in a more economical iterative process: A parametrization is first adjusted to reproduce an initial guess for the value of $a_{\text {surf }}$ or a series of values of $a_{\text {surf }}$ that cover a reasonable range. After convergence of the fit, one calculates then a couple of well-selected fission barriers with the preliminary parametrizations, checks the deviation from data, estimates how $a_{\text {surf }}$ should be changed, and runs a new fit (or series of fits) with the improved estimate for $a_{\text {surf }}$. The process can be repeated until the desired quality for fission barriers is reached.

Figure 5 suggests that the correlation between the surface energy coefficient and the characteristic features of the energy surface is basically the same within each method to calculate $a_{\text {surf }}$, in spite of the HF, ETF, and MTF calculations providing different values for a given parametrization. This means that any of these methods can then be used during the fit as long as the value used for $a_{\text {surf }}$ is tuned accordingly to the formalism used.

Because of its computational simplicity, we use the MTF value for $a_{\text {surf }}$ to construct a series of fits with a surface energy coefficient systematically varied in the range between 18.0 and 19.4 MeV in steps of $0.2 \mathrm{MeV}$, while everything else in the fit protocol is kept unchanged. As a starting point, we chose the protocol used to adjust SLy5* [70], which differs from the fit protocol of the original SLy5 parametrization [69] mainly by an additional constraint that avoids the appearance of unphysical finite-size instabilities in the spin channels [70]. To have a series of parametrizations with similar bulk properties, we have added an additional constraint on the value of the $L$

TABLE I. Nuclear-matter properties of the SLy5s $X$ Skyrme EDFs adjusted for this work. The properties of the original SLy5 [69] and SLy5* [70] parametrizations are also given for comparison. The first block (columns 2-8) shows the standard bulk properties for infinite symmetric matter, i.e., saturation density $\rho_{\text {sat }}$ in $\mathrm{fm}^{-3}$, energy per particle $E / A$ in $\mathrm{MeV}$, incompressibility $K_{\infty}$ in $\mathrm{MeV}$, isoscalar effective mass $m_{0}^{*} / m$, symmetry energy coefficient $J$ and its slope $L$ in MeV, and enhancement factor of the TRK sum rule $\kappa_{v}$. The second block (semibulk; columns 9-11) shows surface energy coefficients $a_{\text {surf }}$ in MeV as obtained from semi-infinite nuclear matter calculations within the MTF, HF, and ETF schemes (see text). The third block (column 12) shows $a_{\text {surf }}$ as deduced from ETF calculations of large finite nuclei.

\begin{tabular}{|c|c|c|c|c|c|c|c|c|c|c|c|}
\hline Model & $\rho_{0}$ & $E / A$ & $K_{\infty}$ & $m^{*} / m$ & $J$ & $L$ & $\kappa_{v}$ & $a_{\text {surf }}^{(\mathrm{MTF})}$ & $a_{\text {surf }}^{(\mathrm{HF})}$ & $a_{\text {surf }}^{(\mathrm{ETF})}$ & $\begin{array}{l}\text { From finite nuclei } \\
\qquad a_{\text {surf }}^{(\mathrm{ETF})}\end{array}$ \\
\hline SLy5* & 0.1605 & -16.02 & 229.9 & 0.7006 & 32.01 & 45.9 & 0.4181 & 19.15 & 18.61 & 18.27 & 18.28 \\
\hline SLy5s1 & 0.1598 & -15.77 & 222.1 & 0.7392 & 31.43 & 48.1 & 0.3047 & 18.00 & 17.55 & 17.15 & 17.16 \\
\hline SLy5s2 & 0.1603 & -15.82 & 223.2 & 0.7350 & 31.60 & 48.3 & 0.3063 & 18.20 & 17.74 & 17.34 & 17.35 \\
\hline SLy5s5 & 0.1618 & -15.96 & 226.4 & 0.7243 & 32.11 & 48.6 & 0.3131 & 18.80 & 18.31 & 17.92 & 17.93 \\
\hline SLy5s6 & 0.1623 & -16.01 & 227.3 & 0.7217 & 32.29 & 48.8 & 0.3160 & 19.00 & 18.50 & 18.11 & 18.13 \\
\hline SLy5s7 & 0.1629 & -16.05 & 228.3 & 0.7196 & 32.46 & 48.9 & 0.3191 & 19.20 & 18.70 & 18.31 & 18.32 \\
\hline SLy5s8 & 0.1634 & -16.10 & 229.1 & 0.7178 & 32.64 & 49.0 & 0.3225 & 19.40 & 18.89 & 18.50 & 18.52 \\
\hline
\end{tabular}




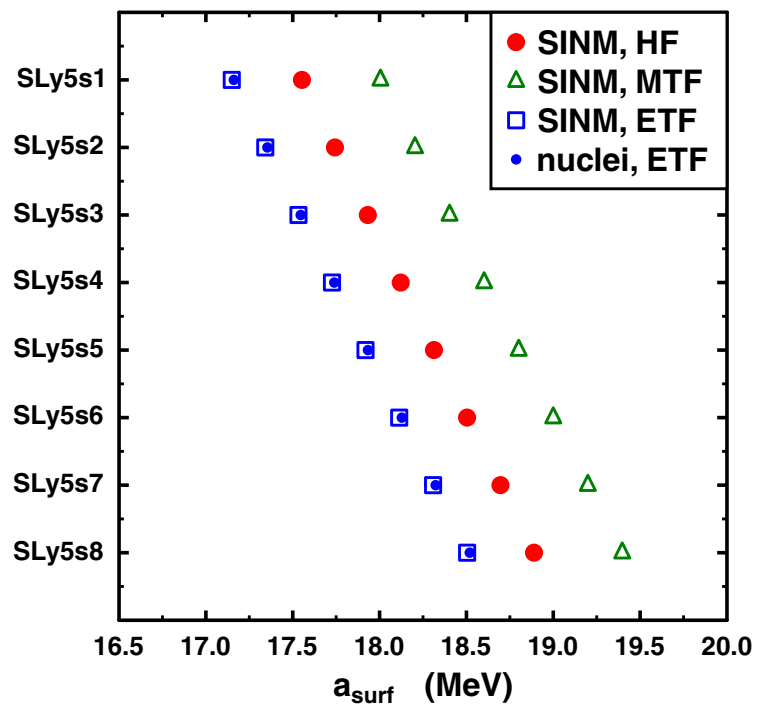

FIG. 7. Surface-energy coefficients $a_{\text {surf }}$ of the SLy5s $X$ parametrizations as obtained from HF, ETF, and MTF calculations of semi-infinite nuclear matter as well as ETF calculations of finite nuclei as described in Sec. III.

coefficient of nuclear matter, which otherwise is only scarcely constrained by finite nuclei, such that it remains in the interval of $(50 \pm 2) \mathrm{MeV}$.

The resulting parametrizations are called SLy5s $X, X=$ $1, \ldots, 8$. Their nuclear-matter properties are summarized in Table I. When comparing any two parametrizations, the difference between the values of the surface energy coefficients obtained with different methods is almost constant; see Fig. 7.

Figure 8 displays the deformation energy along the static fission path of ${ }^{240} \mathrm{Pu}$ obtained with these parametrizations. As

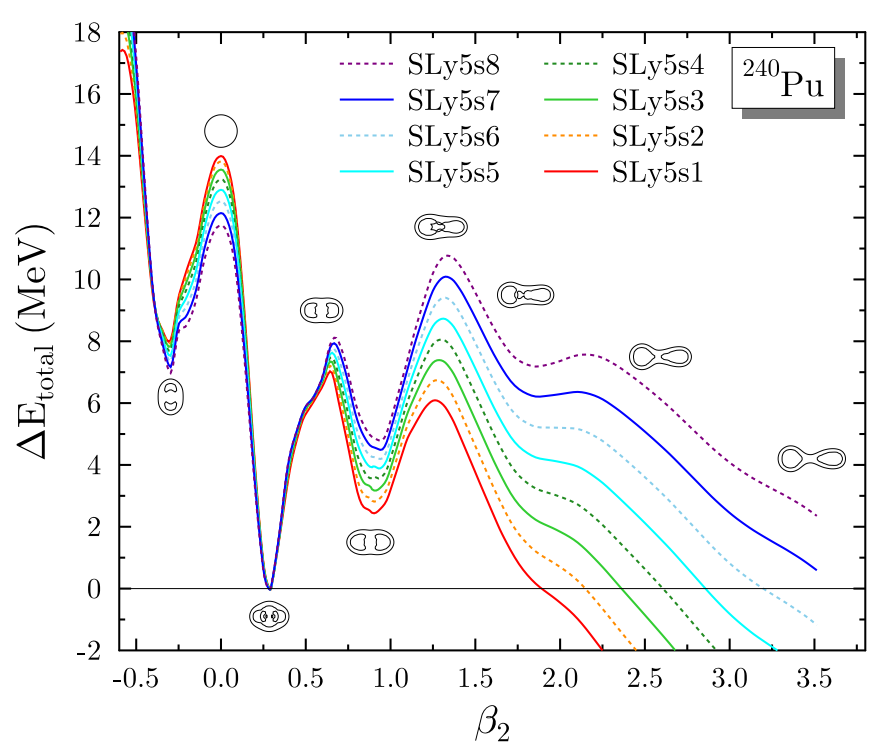

FIG. 8. Same as Fig. 6, but for the family of SLy5s $X$ fits. The insets show contour plots of the mass density distribution in the $x-z$ plane at selected deformations.

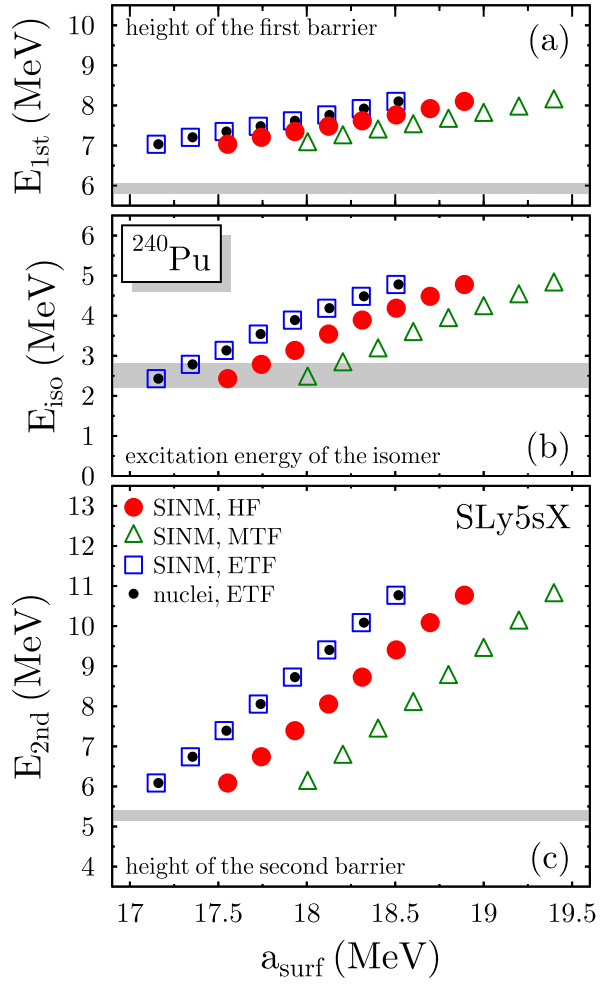

FIG. 9. Correlation between the excitation energy of the fission isomer (a), the height of the inner (b) and outer (c) barriers of ${ }^{240} \mathrm{Pu}$, and the surface energy coefficient $a_{\text {surf }}$ calculated in HF, ETF, and MTF for the SLy5sx series of interactions. The horizontal gray bars indicate the range of experimental data found in the literature (see text). The energy scales and intervals are the same as in Fig. 5.

they are all fitted within the same protocol but for the value that $a_{\text {surf }}$ is fixed at, the evolution of the curves with $a_{\text {surf }}$ is now much more regular. However, there are still indications that also shell effects are slowly varying in response to the change of $a_{\text {surf }}$ in the fit protocol: At $\beta_{2}$ values around 1.7, a shallow third minimum develops with increasing value of $a_{\text {surf }}$.

Still, for the excitation energies of the fission isomer and the inner and outer saddles, the correlation with $a_{\text {surf }}$ is now almost perfectly linear, as demonstrated by Fig. 9. Still, none of the parametrizations does simultaneously describe all of the three properties.

In the present study, we have focused on the isoscalar surface energy coefficient $a_{\text {surf }}$. All candidates for heavy nuclei whose deformation energy can be used to constrain parameter fits, however, have a neutron excess. For example, the nucleus ${ }^{240} \mathrm{Pu}$ used as a benchmark above has an asymmetry of $I \equiv(N-Z) /(N+Z)=0.22$. The leptodermous expansion of the nuclear binding energy suggests that there is a correction to the surface energy that depends explicitly on the nucleus' asymmetry $I$ and that in the liquid-drop model is parametrized through the surface symmetry energy coefficient $a_{\text {ssym }}[22,33,62,63]$.

If a single nucleus is used to constrain the surface energy, there is the danger that $a_{\text {ssym }}$ accidentally takes a wrong 
value and thereby introduces an erroneous isospin dependence of fission barriers. However, as demonstrated in a detailed analysis of this quantity's role for the systematics of the excitation energy of superdeformed states of heavy nuclei [22], the value of the $a_{\text {ssym }}$ of existing parametrizations of the Skyrme EDF is strongly correlated with the volume energy coefficient and therefore fixed by masses along the valley of stability, such that it cannot vary freely over a wide interval. This gives us confidence that $a_{\text {ssym }}$ takes a reasonable and consistent value for all SLy5s $X$ fits. Indeed, for all of the SLy $5 \mathrm{~s} X$ parametrizations we find values of $a_{\text {ssym }}$ that are close to $-49 \mathrm{MeV}$. Also, calculations of the fission barriers of other heavy nuclei that will be reported elsewhere indicate that the overall trend of the predicted fission barriers does not change with asymmetry. For example, when calculated with SLy5s1, the fission barrier height of the much less asymmetric nucleus ${ }^{180} \mathrm{Hg}$ with $I=0.11$ is described as satisfactorily as the barrier of ${ }^{240} \mathrm{Pu}$.

\section{DISCUSSION AND CONCLUSIONS}

We summarize here our main findings concerning the various methods to calculate $a_{\text {surf }}$.

(i) HF, ETF, and MTF calculations for semi-infinite nuclear matter provide very consistent, but not identical, values for $a_{\text {surf }}$. The MTF method gives always slightly larger values than the HF approach, whereas the ETF method always gives slightly smaller values than HF. Differences between the values extracted from semiclassical MTF and ETF calculations on the one hand and the quantal HF approach on the other hand are typically on the order of $500 \mathrm{keV}$ out of about $18 \mathrm{MeV}$, but in exceptional cases might be as large as $1 \mathrm{MeV}$.

(ii) The values of $a_{\text {surf }}$ as extracted from ETF calculations of semi-infinite nuclear matter and from the systematics of the ETF binding energies of very large artificial spherical nuclei are very close and differ rarely by more than $10 \mathrm{keV}$. To reach this level of agreement, an $A^{0}$ term has to be included in the leptodermous expansion of the binding energy of finite nuclei and the contribution of the center-of-mass correction omitted.

(iii) For the purpose of calculating $a_{\text {surf }}$, the MTF and ETF approximations are fairly robust. The deviation from $\mathrm{HF}$ values is reasonably parametrization independent, although there are differences in detail that are correlated to the presence and strength of the tensor terms, the incompressibility $K_{\infty}$, and the isoscalar effective mass $m_{0}^{*} / m$. During a fit, the values for $K_{\infty}, m_{0}^{*} / m$, and the coupling constants of the tensor terms will rarely vary over a large interval, such that the analytical MTF value for $a_{\text {surf }}$ as obtained from Eq. (22) can be safely used in a fit protocol to determine the coupling constants of Skyrme EDFs. In any event, we do not aim to reproduce a universal empirical value for $a_{\text {surf }}$, which will be difficult to extract in a model-independent way from data anyway, but instead provide a simple and efficient control over the surface properties within a given framework.

Concerning the correlation between characteristic energies of the fission barrier of ${ }^{240} \mathrm{Pu}$ and the values for $a_{\text {surf }}$ we find the following.

(i) The simultaneous description of the fission barrier heights and the excitation energy of the fission isomer is not trivial because they are also strongly sensitive to shell effects which are at the very origin of the complicated topography of the deformation energy surface.

(ii) While the characteristic energies of fission barriers are clearly and unambiguously correlated to the surface energy coefficient in the expected manner, for existing Skyrme parametrizations there is a large scatter when plotting them as a function of the surface energy coefficient. This is a consequence of the shell effects being unsystematically different when parametrizations are constructed with different fit protocols.

To eliminate the protocol-dependence of the analysis of $a_{\text {surf }}$ and its correlation with fission barriers, and as a proof of principle for a parameter fit that includes the MTF value for $a_{\text {surf }}$ in its protocol, we have constructed a series of parametrizations of the standard Skyrme functional called SLy5s1, SLy5s2,.., SLy5s8, with systematically varied surface energy coefficients.

(i) The resulting parametrizations exhibit almost linear correlations between the surface energy coefficient on the one hand and each of the characteristic energies of the energy surface of ${ }^{240} \mathrm{Pu}$ on the other hand, providing the proof-of-principle for replacing the adjustment of fission barriers with a suitably chosen value of $a_{\text {surf }}$ as obtained from semi-infinite nuclear-matter calculations with any of the schemes considered here.

(ii) None of the SLy5s $X$ parametrizations, however, reproduces simultaneously the empirical data for the excitation energy of the fission isomer as well as the heights of the inner and outer barriers. This points to imperfections of the deformation dependence of shell effects provided by these parametrizations.

(iii) The best reproduction of the energy surface of ${ }^{240} \mathrm{Pu}$ is provided by SLy5s1, the parametrization with the lowest value of $a_{\text {surf }}$ considered in the series of fits.

Adjusting parameters to reproduce a value for $a_{\text {surf }}$ is computationally much easier than reproducing fission barriers of heavy nuclei, not only with respect to CPU time, but, even more importantly, also in terms of stability and controllability of the calculations during the early stages of a fit when the parameters are still far from their physical values such that the topography of the energy surfaces might be very different from the physical one. In practice, however, the appropriate value of $a_{\text {surf }}$ that provides the best description of the targeted deformation energies will have to be determined iteratively, alternating between complete parameter fits for some guess(es) 
for $a_{\text {surf }}$ on the one hand, and the calculation of deformation energy surfaces with the so-determined parametrizations on the other hand. The latter then provide a refined guess for $a_{\text {surf }}$, if necessary. In the present paper, we limited ourselves to the first of such cycles.

As many of the widely used Skyrme parametrizations provide an unsatisfactory description of the systematics of fission barriers and excitation energies of very deformed states $[1,22,101,111]$, it is highly desirable to better constrain the nuclear surface energy in future fits. Besides the obvious importance for the study and understanding of the fission process itself, fission barrier heights are also a determining factor for the stability of superheavy nuclei [101,111] and the dynamics of the astrophysical $r$ process of nucleosynthesis [21,49,111].

In conclusion, the use of the MTF pocket formula for $a_{\text {surf }}$ provides a rapid and robust expression to control the surface properties of nuclear energy density functionals during the adjustment of their parameter. In this paper, we have focused on the (isoscalar) surface energy coefficient. Within the MTF approach, the integral that appears in the calculation of the (isovector) surface symmetry energy coefficient from semi-infinite matter cannot be solved analytically. However, making further approximations whose consequences remain to be analyzed, one can arrive at an analytical expression for this quantity as well [112], which might offer a route to its efficient fine tuning.

The generalization of the MTF pocket formula to Skyrmetype functionals with derivative terms of order 4 or 6 [13-15] is feasible, but will require an arduous extension of the formalism to higher-order terms.

\section{ACKNOWLEDGMENTS}

Stimulating discussions with D. Davesne about semiclassical methods in physics are gratefully acknowledged. We also thank W. Ryssens for critical reading of an early version of the manuscript. This work was supported by the Agence Nationale de la Recherche under Grant No. ANR 2010 BLANC 0407 "NESQ," by the CNRS/IN2P3 through PICS Grant No. 6949, and by the Academy of Finland and University of Jyväskylä within the FIDIPRO program. Part of the computations were performed using HPC resources of the MCIA (Mésocentre de Calcul Intensif Aquitain) of the Université de Bordeaux and of the Université de Pau et des Pays de l'Adour.
[1] M. Bender, P.-H. Heenen, and P.-G. Reinhard, Self-consistent mean-field models for nuclear structure, Rev. Mod. Phys. 75 , 121 (2003).

[2] T. H. R. Skyrme, The nuclear surface, Philos. Mag. (17981977) 1, 1043 (1956).

[3] T. H. R. Skyrme, The effective nuclear potential, Nucl. Phys. 9, 615 (1958).

[4] D. Vautherin and D. M. Brink, Hartree-Fock calculations with Skyrme's interaction. I. Spherical nuclei, Phys. Rev. C 5, 626 (1972).

[5] J. Friedrich and P.-G. Reinhard, Skyrme-force parametrization: Least-squares fit to nuclear ground-state properties, Phys. Rev. C 33, 335 (1986).

[6] M. Kortelainen, T. Lesinski, J. Moré, W. Nazarewicz, J. Sarich, N. Schunck, M. V. Stoitsov, and S. Wild, Nuclear energy density optimization, Phys. Rev. C 82, 024313 (2010).

[7] M. Kortelainen, J. McDonnell, W. Nazarewicz, P.-G. Reinhard, J. Sarich, N. Schunck, M. V. Stoitsov, and S. M. Wild, Nuclear energy density optimization: Large deformations, Phys. Rev. C 85, 024304 (2012).

[8] Y. Gao, J. Dobaczewski, M. Kortelainen, J. Toivanen, and D. Tarpanov, Propagation of uncertainties in the Skyrme energydensity-functional model, Phys. Rev. C 87, 034324 (2013).

[9] M. Kortelainen, J. McDonnell, W. Nazarewicz, E. Olsen, P.-G. Reinhard, J. Sarich, N. Schunck, S. M. Wild, D. Davesne, J. Erler, and A. Pastore, Nuclear energy density optimization: Shell structure, Phys. Rev. C 89, 054314 (2014).

[10] J. Dobaczewski, W. Nazarewicz, and P.-G. Reinhard, Error estimates of theoretical models: A guide, J. Phys. G 41, 074001 (2014).

[11] T. Lesinski, M. Bender, K. Bennaceur, T. Duguet, and J. Meyer, Tensor part of the Skyrme energy density functional: Spherical nuclei, Phys. Rev. C 76, 014312 (2007).
[12] B. G. Carlsson, J. Dobaczewski, and M. Kortelainen, Local nuclear energy density functional at next-to-next-to-next-toleading order, Phys. Rev. C 78, 044326 (2008); Erratum: Local nuclear energy density functional at next-to-next-to-next-toleading order [Phys. Rev. C 78, 044326 (2008)], 81, 029904(E) (2010).

[13] F. Raimondi, B. G. Carlsson, and J. Dobaczewski, Effective pseudopotential for energy density functionals with higherorder derivatives, Phys. Rev. C 83, 054311 (2011).

[14] D. Davesne, A. Pastore, and J. Navarro, Skyrme effective pseudopotential up to the next-to-next-to-leading order, J. Phys. G 40, 095104 (2013).

[15] P. Becker, D. Davesne, J. Meyer, A. Pastore, and J. Navarro, Tools for incorporating a D-wave contribution in Skyrme energy density functionals, J. Phys. G 42, 034001 (2015).

[16] J. Sadoudi, M. Bender, K. Bennaceur, D. Davesne, R. Jodon, and T. Duguet, Skyrme pseudo-potential-based EDF parametrization for spuriousity-free MR-EDF calculations, Phys. Scr. T154, 014013 (2013).

[17] J. Sadoudi, T. Duguet, J. Meyer, and M. Bender, Skyrme functional from a three-body pseudopotential of second order in gradients: Formalism for central terms, Phys. Rev. C 88, 064326 (2013).

[18] J. Bartel, P. Quentin, M. Brack, C. Guet, and H.-B. Håkansson, Towards a better parameterization of Skyrme-like effective forces: A critical study of the SkM force, Nucl. Phys. A 386, 79 (1982).

[19] J. Berger, M. Girod, and D. Gogny, Time-dependent quantum collective dynamics applied to nuclear fission, Comp. Phys. Commun. 63, 365 (1991).

[20] M. Bender, K. Rutz, P.-G. Reinhard, and J. A. Maruhn, Consequences of the center-of-mass correction in nuclear mean-field models, Eur. Phys. J. A 7, 467 (2000). 
[21] S. Goriely, M. Samyn, and J. M. Pearson, Further explorations of Skyrme-Hartree-Fock-Bogoliubov mass formulas. VII. Simultaneous fits to masses and fission barriers, Phys. Rev. C 75, 064312 (2007).

[22] N. Nikolov, N. Schunck, W. Nazarewicz, M. Bender, and J. Pei, Surface symmetry energy of nuclear energy density functionals, Phys. Rev. C 83, 034305 (2011).

[23] M. Beiner, H. Flocard, Nguyen Van Giai, and P. Quentin, Nuclear ground-state properties and self-consistent calculations with the Skyrme interaction: (I). Spherical description, Nucl. Phys. A 238, 29 (1975).

[24] H. Krivine, J. Treiner, and O. Bohigas, Derivation of a fluid-dynamical lagrangian and electric giant resonances, Nucl. Phys. A 336, 155 (1980).

[25] M. Brack, C. Guet, and H.-B. Håkansson, Selfconsistent semiclassical description of average nuclear properties - a link between microscopic and macroscopic models, Phys. Rep. 123, 275 (1985).

[26] L. Bonneau, P. Quentin, and D. Samœn, Fission barriers of heavy nuclei within a microscopic approach, Eur. Phys. J. A 21, 391 (2005).

[27] A. Staszczak, A. Baran, and W. Nazarewicz, Spontaneous fission modes and lifetimes of superheavy elements in the nuclear density functional theory, Phys. Rev. C 87, 024320 (2013).

[28] N. Schunck, D. Duke, H. Carr, and A. Knoll, Description of induced nuclear fission with Skyrme energy functionals: Static potential energy surfaces and fission fragment properties, Phys. Rev. C 90, 054305 (2014).

[29] J. Sadhukhan, K. Mazurek, A. Baran, J. Dobaczewski, W. Nazarewicz, and J. A. Sheikh, Spontaneous fission lifetimes from the minimization of self-consistent collective action, Phys. Rev. C 88, 064314 (2013).

[30] J. Sadhukhan, J. Dobaczewski, W. Nazarewicz, J. A. Sheikh, and A. Baran, Pairing-induced speedup of nuclear spontaneous fission, Phys. Rev. C 90, 061304 (2014).

[31] J. D. McDonnell, W. Nazarewicz, J. A. Sheikh, A. Staszczak, and M. Warda, Excitation-energy dependence of fission in the mercury region, Phys. Rev. C 90, 021302 (2014).

[32] J. Côté and J. M. Pearson, Hartree-Fock calculations of semiinfinite nuclear matter with complete forces (finite-range and spin-orbit term), Nucl. Phys. A 304, 104 (1978).

[33] P.-G. Reinhard, M. Bender, W. Nazarewicz, and T. Vertse, From finite nuclei to the nuclear liquid drop: Leptodermous expansion based on self-consistent mean-field theory, Phys. Rev. C 73, 014309 (2006).

[34] W. Ryssens, V. Hellemans, M. Bender, and P.-H. Heenen, Solution of the Skyrme HF+BCS equation on a 3D mesh II. A new version of the Ev8 code, Com. Phys. Commun. 187, 175 (2015).

[35] J. Sadoudi, Constraints on the nuclear energy density functional and new possible analytical forms, Ph.D. thesis, Université Paris-Sud XI, 2011.

[36] E. Perlińska, S. G. Rohoziński, J. Dobaczewski, and W. Nazarewicz, Local density approximation for proton-neutron pairing correlations: Formalism, Phys. Rev. C 69, 014316 (2004).

[37] W. J. Swiatecki, The nuclear surface energy, Proc. Phys. Soc. A 64, 226 (1951).

[38] W. D. Myers and W. J. Swiatecki, Droplet model theory of the neutron skin, Nucl. Phys. A 336, 267 (1980).
[39] W. D. Myers, W. J. Swiatecki, and C. S. Wang, The nuclear surface of multi-component systems, Nucl. Phys. A 436, 185 (1985).

[40] M. Farine, J. Côté, and J. M. Pearson, Hartree-Fock calculations of surface-symmetry properties with finite-range forces, Nucl. Phys. A 338, 86 (1980).

[41] M. Farine and J. M. Pearson, On the two definitions of nuclear surface energy and its calculation in two-component systems, Phys. Lett. B 167, 259 (1986).

[42] K. Kolehmainen, M. Prakash, J. M. Lattimer, and J. R. Treiner, Surface and curvature properties of neutron-rich nuclei, Nucl. Phys. A 439, 535 (1985).

[43] M. Centelles, M. Del Estal, and X. Viñas, Semiclassical treatment of asymmetric semi-infinite nuclear matter: Surface and curvature properties in relativistic and non-relativistic models, Nucl. Phys. A 635, 193 (1998).

[44] F. Douchin, P. Haensel, and J. Meyer, Nuclear surface and curvature properties for SLy Skyrme forces and nuclei in the inner neutron-star crust, Nucl. Phys. A 665, 419 (2000).

[45] J. Friedel, Metallic alloys, Il Nuovo Cimento, Suppl. 7, 287 (1958).

[46] A. Ayachi, M. Durand, P. Schuck, and V. S. Ramamurthy, Phase space study of Friedel oscillations close to the surface of half infinite nuclear matter, Z. Phys. A 327, 141 (1987).

[47] M. Farine, J. Côté, and J. M. Pearson, Surface coefficients of Skyrme-Orsay forces, Phys. Rev. C 24, 303 (1981).

[48] M. Farine, J. M. Pearson, and F. Tondeur, Nuclearmatter incompressibility from fits of generalized Skyrme force to breathing-mode energies, Nucl. Phys. A 615, 135 (1997).

[49] M. Samyn, S. Goriely, and J. M. Pearson, Further explorations of Skyrme-Hartree-Fock-Bogoliubov mass formulas. V. Extension to fission barriers, Phys. Rev. C 72, 044316 (2005).

[50] M. Samyn (private communication), as quoted in Refs. [22] and [33].

[51] P. Danielewicz and J. Lee, Symmetry energy I: Semi-infinite matter, Nucl. Phys. A 818, 36 (2009).

[52] See Supplemental Material at http://link.aps.org/ supplemental/10.1103/PhysRevC.94.024335 for tables with values used to prepare the figures, coupling constants of the SLy $5 \mathrm{~s} X$ parametrizations, and other details.

[53] M. Brack, in Atomic clusters and nanoparticles, Agregats atomiques et nanoparticules, edited by C. Guet, P. Hobza, F. Speigelman, and F. David, Les Houches-École d'Été de Physique Théorique, Session LXXIII (Springer, Berlin, Heidelberg, 2001), Chap. 5, p. 161.

[54] J. Bartel and K. Bencheikh, Nuclear mean fields through selfconsistent semiclassical calculations, Eur. Phys. J. A 14, 179 (2002).

[55] J. Bartel, K. Bencheikh, and J. Meyer, Extended Thomas-Fermi density functionals in the presence of a tensor interaction in spherical symmetry, Phys. Rev. C 77, 024311 (2008).

[56] H. Krivine and J. Treiner, A simple approximation to the nuclear kinetic energy density, Phys. Lett. B 88, 212 (1979).

[57] J. Treiner and H. Krivine, Semi-classical nuclear properties from effective interactions, Ann. Phys. (N.Y.) 170, 406 (1986).

[58] R. A. Berg and L. Wilets, Nuclear surface effects, Phys. Rev. 101, 201 (1956). 
[59] L. Wilets, Neutron and proton densities in nuclei, Phys. Rev. 101, 1805 (1956).

[60] J. Margueron, J. Navarro, and N. V. Giai, Instabilities of infinite matter with effective Skyrme-type interactions, Phys. Rev. C 66, 014303 (2002).

[61] B. K. Agrawal, S. Shlomo, and V. K. Au, Determination of the parameters of a Skyrme type effective interaction using the simulated annealing approach, Phys. Rev. C 72, 014310 (2005).

[62] K. Pomorski and J. Dudek, Nuclear liquid-drop model and surface-curvature effects, Phys. Rev. C 67, 044316 (2003).

[63] G. Royer and C. Gautier, Coefficients and terms of the liquid drop model and mass formula, Phys. Rev. C 73, 067302 (2006).

[64] K. T. R. Davies and J. R. Nix, Calculation of moments, potentials, and energies for an arbitrarily shaped diffusesurface nuclear density distribution, Phys. Rev. C 14, 1977 (1976).

[65] H. J. Krappe, J. R. Nix, and A. J. Sierk, Unified nuclear potential for heavy-ion elastic scattering, fusion, fission, and ground-state masses and deformations, Phys. Rev. C 20, 992 (1979).

[66] P. Möller, J. R. Nix, W. D. Myers, and W. J. Swiatecki, Nuclear ground-state masses and deformations, Atom. Data Nucl. Data Tables 59, 185 (1995).

[67] H. S. Köhler, Nuclear masses, shell-model and superheavy nuclei (I), Nucl. Phys. A 162, 385 (1971).

[68] N. V. Giai and H. Sagawa, Spin-isospin and pairing properties of modified Skyrme interactions, Phys. Lett. B 106, 379 (1981).

[69] E. Chabanat, P. Bonche, P. Haensel, J. Meyer, and R. Schaeffer, A Skyrme parametrization from subnuclear to neutron star densities. Part II. Nuclei far from stabilities, Nucl. Phys. A 635, 231 (1998); Erratum: A Skyrme parametrization from subnuclear to neutron star densities. (II): Nuclei far from stabilities [Nucl. Phys. A 635 (1998) 231-256], 643, 441(E) (1998).

[70] A. Pastore, D. Davesne, K. Bennaceur, J. Meyer, and V. Hellemans, Fitting Skyrme functionals using linear response theory, Phys. Scr. T154, 014014 (2013).

[71] K.-H. Kim, T. Otsuka, and P. Bonche, Three-dimensional TDHF calculations for reactions of unstable nuclei, J. Phys. G 23, 1267 (1997).

[72] G. Colò, H. Sagawa, S. Fracasso, and P. F. Bortignon, Spin-orbit splitting and the tensor component of the Skyrme interaction, Phys. Lett. B 646, 227 (2007); Erratum to: "Spin-orbit splitting and the tensor component of the Skyrme interaction" [Phys. Lett. B 646 (2007) 227], 668, 457(E) (2008).

[73] M. Zalewski, J. Dobaczewski, W. Satuła, and T. R. Werner, Spin-orbit and tensor mean-field effects on spin-orbit splitting including self-consistent core polarizations, Phys. Rev. C 77, 024316 (2008).

[74] M. Bender, K. Bennaceur, T. Duguet, P. H. Heenen, T. Lesinski, and J. Meyer, Tensor part of the Skyrme energy density functional. II. Deformation properties of magic and semi-magic nuclei, Phys. Rev. C 80, 064302 (2009).

[75] T. Lesinski, K. Bennaceur, T. Duguet, and J. Meyer, Isovector splitting of nucleon effective masses, ab initio benchmarks and extended stability criteria for Skyrme energy functionals, Phys. Rev. C 74, 044315 (2006).
[76] E. Chabanat, P. Bonche, P. Haensel, J. Meyer, and R. Schaeffer, A Skyrme parametrization from subnuclear to neutron star densities, Nucl. Phys. A 627, 710 (1997).

[77] K. Washiyama, K. Bennaceur, B. Avez, M. Bender, P.-H. Heenen, and V. Hellemans, New parametrization of Skyrme's interaction for regularized multireference energy density functional calculations, Phys. Rev. C 86, 054309 (2012).

[78] X. Roca-Maza, G. Colò, and H. Sagawa, New Skyrme interaction with improved spin-isospin properties, Phys. Rev. C 86, 031306 (2012).

[79] P.-G. Reinhard and H. Flocard, Nuclear effective forces and isotope shifts, Nucl. Phys. A 584, 467 (1995).

[80] P.-G. Reinhard, D. J. Dean, W. Nazarewicz, J. Dobaczewski, J. A. Maruhn, and M. R. Strayer, Shape coexistence and the effective nucleon-nucleon interaction, Phys. Rev. C 60, 014316 (1999).

[81] P. Klüpfel, P.-G. Reinhard, T. J. Bürvenich, and J. A. Maruhn, Variations on a theme by Skyrme: A systematic study of adjustments of model parameters, Phys. Rev. C 79, 034310 (2009).

[82] Y. Shi, J. Dobaczewski, and P. T. Greenlees, Rotational properties of nuclei around ${ }^{254}$ No investigated using a spectroscopicquality Skyrme energy density functional, Phys. Rev. C 89, 034309 (2014).

[83] M. Samyn, S. Goriely, and J. Pearson, Further explorations of Skyrme-Hartree-Fock-Bogoliubov mass formulas. I: Role of density dependence in pairing force, Nucl. Phys. A 725, 69 (2003).

[84] S. Goriely, M. Samyn, M. Bender, and J. M. Pearson, Further explorations of Skyrme-Hartree-Fock-Bogoliubov mass formulas. II. Role of the effective mass, Phys. Rev. C 68, 054325 (2003).

[85] M. Samyn, S. Goriely, M. Bender, and J. M. Pearson, Further explorations of Skyrme-Hartree-Fock-Bogoliubov mass formulas. III. Role of particle-number projection, Phys. Rev. C 70, 044309 (2004).

[86] S. Goriely, M. Samyn, J. M. Pearson, and M. Onsi, Further explorations of Skyrme-Hartree-Fock-Bogoliubov mass formulas. IV: Neutron-matter constraint, Nucl. Phys. A 750, 425 (2005).

[87] S. Goriely, M. Samyn, and J. Pearson, Further explorations of Skyrme-Hartree-Fock-Bogoliubov mass formulas. VI: Weakened pairing, Nucl. Phys. A 773, 279 (2006).

[88] S. Goriely and J. M. Pearson, Further explorations of Skyrme-Hartree-Fock-Bogoliubov mass formulas. VIII. Role of Coulomb exchange, Phys. Rev. C 77, 031301 (2008).

[89] L. G. Cao, U. Lombardo, C. W. Shen, and N. V. Giai, From Brueckner approach to Skyrme-type energy density functional, Phys. Rev. C 73, 014313 (2006).

[90] A. Steiner, M. Prakash, J. Lattimer, and P. Ellis, Isospin asymmetry in nuclei and neutron stars, Phys. Rep. 411, 325 (2005).

[91] P. D. Stevenson, P. M. Goddard, J. R. Stone, and M. Dutra, Do Skyrme forces that fit nuclear matter work well in finite nuclei's, AIP Conf. Proc. 1529, 262 (2013).

[92] P. Guichon, H. Matevosyan, N. Sandulescu, and A. Thomas, Physical origin of density dependent forces of Skyrme type within the quark meson coupling model, Nucl. Phys. A 772, 1 (2006).

[93] J. Dobaczewski, Ab initio derivation of model energy density functionals, J. Phys. G 43, 04LT01 (2016). 
[94] R. Jodon, Constraints on the nuclear energy density functional and new possible analytical forms, Ph.D. thesis, Université Lyon 1, 2014.

[95] M. Dutra, O. Lourenço, J. S. Sá Martins, A. Delfino, J. R. Stone, and P. D. Stevenson, Skyrme interaction and nuclear matter constraints, Phys. Rev. C 85, 035201 (2012).

[96] S. Bjørnholm and J. E. Lynn, The double-humped fission barrier, Rev. Mod. Phys. 52, 725 (1980).

[97] M. Brack, J. Damgaard, A. S. Jensen, H.-C. Pauli, V. M. Strutinsky, and C. Y. Wong, Funny hills: The shell-correction approach to nuclear shell effects and its applications to the fission process, Rev. Mod. Phys. 44, 320 (1972).

[98] F. Tondeur, Fission barriers and the parametrisation of Skyrme forces, Nucl. Phys. A 442, 460 (1985).

[99] M. Bender, P.-H. Heenen, and P. Bonche, Microscopic study of ${ }^{240} \mathrm{Pu}$ : Mean field and beyond, Phys. Rev. C 70, 054304 (2004).

[100] K. Rutz, J. A. Maruhn, P.-G. Reinhard, and W. Greiner, Fission barriers and asymmetric ground states in the relativistic meanfield theory, Nucl. Phys. A 590, 680 (1995).

[101] T. Bürvenich, M. Bender, J. A. Maruhn, and P.-G. Reinhard, Systematics of fission barriers in superheavy elements, Phys. Rev. C 69, 014307 (2004).

[102] W. Younes and D. Gogny, Microscopic calculation of ${ }^{240} \mathrm{Pu}$ scission with a finite-range effective force, Phys. Rev. C 80, 054313 (2009).

[103] C. Rigollet, P. Bonche, H. Flocard, and P.-H. Heenen, Microscopic study of the properties of identical bands in the $A=150$ mass region, Phys. Rev. C 59, 3120 (1999).
[104] P. Bonche, P.-H. Heenen, M. Bender, and W. Ryssens, EV4 $\mathrm{HF}+\mathrm{BCS}$ code (unpublished).

[105] W. Ryssens, P.-H. Heenen, and M. Bender, Numerical accuracy of mean-field calculations in coordinate space, Phys. Rev. C 92, 064318 (2015).

[106] B. Singh, R. Zywina, and R. Firestone, Table of superdeformed nuclear bands and fission isomers: Third edition, Nucl. Data Sheets 97, 241 (2002).

[107] M. Hunyadi, D. Gassmann, A. Krasznahorkay, D. Habs, G. Thirolf, M. Csatlós, Y. Eisermann, T. Faestermann, G. Graw, J. Gulyas, R. Hertenberger, H. Maier, Z. Mátáa, A. Metz, and M. Chromik, Excited superdeformed $K^{\pi}=0^{+}$rotational bands in $\beta$-vibrational fission resonances of ${ }^{240} \mathrm{Pu}$, Phys. Lett. B 505, 27 (2001).

[108] A. Mamdouh, J. Pearson, M. Rayet, and F. Tondeur, Largescale fission-barrier calculations with the ETFSI method, Nucl. Phys. A 644, 389 (1998).

[109] R. Capote et al., RIPL - reference input parameter library for calculation of nuclear reactions and nuclear data evaluations, Nucl. Data Sheets 110, 3107 (2009), special Issue on Nuclear Reaction Data.

[110] G. N. Smirenkin, Preparation of evaluated data for a fission barrier parameter library for isotopes with $Z=82-98$, with consideration of the level density models used, IAEA-Report INDC(CCP)-359, Tech. Rep., IAEA, Vienna, 1993.

[111] J. Erler, K. Langanke, H. P. Loens, G. Martínez-Pinedo, and P.-G. Reinhard, Fission properties for $r$-process nuclei, Phys. Rev. C 85, 025802 (2012).

[112] H. Krivine and J. Treiner, A simple determination of the nuclear surface symmetry energy, Phys. Lett. B 124, 127 (1983). 